\title{
Numerical methods for scattering problems in periodic waveguides
}

\author{
Ruming Zhang ${ }^{1}$
}

Received: 24 March 2020 / Revised: 14 June 2021 / Accepted: 30 July 2021

(c) The Author(s) 2021

\begin{abstract}
In this paper, we propose new numerical methods for scattering problems in periodic waveguides. Based on [20], the "physically meaningful" solution, which is obtained via the Limiting Absorption Principle (LAP) and is called an LAP solution, is written as an integral of quasi-periodic solutions on a contour. The definition of the contour depends both on the wavenumber and the periodic structure. The contour integral is then written as the combination of finite propagation modes and a contour integral on a small circle. Numerical methods are developed and based on the two representations. Compared with other numerical methods, we do not need the LAP process during numerical approximations, thus a standard error estimation is easily carried out. Based on this method, we also develop a numerical solver for halfguide problems. The method is based on the result that any LAP solution of a halfguide problem can be extended to the LAP solution of a fullguide problem. At the end of this paper, we also give some numerical results to show the efficiency of our numerical methods.
\end{abstract}

Keywords Scattering problems $\cdot$ Periodic waveguide $\cdot$ Limiting absorption principle $\cdot$ Curve integration $\cdot$ Finite element method

Mathematics Subject Classification 35P25 $\cdot 35 \mathrm{~A} 35 \cdot 65 \mathrm{M} 60$

\section{Introduction}

Numerical simulation for scattering problems in periodic waveguides is an interesting topic in both mathematics and related technologies, due to its wide applications in optics, nanotechnology, etc. As the well-posedness of the scattering problems is not

Ruming Zhang

ruming.zhang@kit.edu

1 Institute of Applied and Numerical mathematics, Karlsruhe Institute of Technology, Karlsruhe, Germany 
always true, the Limiting Absorption Principle (LAP) is commonly used to find out the "physically meaningful" solution. That is, the "physically meaningful solution" is assumed to be the limit of a family of solutions with absorbing material, when the positive absorption parameter tends to 0 . In this paper, the limit is called the LAP solution. It has been proved that LAP solutions exist for planar waveguides filled up with periodic material, and we refer to [1-4] for different proofs.

In recent years, some numerical methods have been developed to solve this kind of problems based on the LAP. As the problem with absorbing medium is uniquely solvable and the unique solution decays exponentially along the periodic waveguide, the solution is easily approximated by problems defined in bounded domains and then computed by standard numerical methods. We refer to [6,23] for a numerical method with the help of a so-called recursive doubling process to approximate Robinto-Robin maps on left-and right-boundaries of a periodic cell, and the original solution is approximated by the one with a sufficiently small positive absorption parameter. Based on this idea, the solution is also approximated by an extrapolation technique with respect to small positive absorption parameters in [5], and the method is also extended to scattering problems in locally perturbed periodic layers in [7]. On the other hand, the LAP has also been applied to pick up "proper" propagation modes. With the asymptotic behaviour of propagating modes when the absorption parameter tends to 0 , it is possible to determine whether certain modes propagate to the left or to the right. With these propagating modes, Dirichlet-to-Neumann maps on the boundaries of periodic cells are approximated and the solution for the whole problem is computed. For details we refer to $[8,15]$. For other numerical methods we also refer to [16-18].

Recently, the Floquet-Bloch transform has been applied to both theoretical analysis and numerical simulation for scattering problems in periodic structures. We refer to $[9,10,19]$ for scattering problems with (perturbed) periodic media, to [11-14] for problems with periodic surfaces, and to [2-4] for periodic waveguides. From the Floquet-Bloch theory, the unique solution of the periodic waveguide problem with absorption is written as a contour integral on the unit circle, where the integrand is a family of quasi-periodic solutions depending analytically on the quasi-periodicities. When the absorbing parameter tends to 0 , the integrand may become irregular as poles may approach the unit circle during this process. Based on $[1,8,15]$, we replace the unit circle by a small modification of it. For the choice of the new curve, we require that the integral does not change for any sufficiently small absorbing parameter, and the integrand does not have any poles on the new curve when the parameter is 0 . We also show that when the wavenumber satisfies certain conditions, such a curve always exists. Luckily, we have proved that the wavenumbers such that the conditions are not satisfied compose a discrete subset of $\mathbb{R}_{+}$. Thus for any positive wavenumber except for this discrete set, we can write the LAP solution as a contour integral on a closed curve, where the integrand is a family of quasi-periodic solutions of cell problems. As the quasi-periodic cell problems are classical, the numerical simulation of LAP solutions is also easily carried out. The curve can be easily chosen as a piecewise analytic one, as there are only finite number of eigenvalues on the unit circle. Finally, as the solution depends piecewise analytically on the curve, a high order numerical method is designed based on the contour integral. Moreover, we can also extend this 


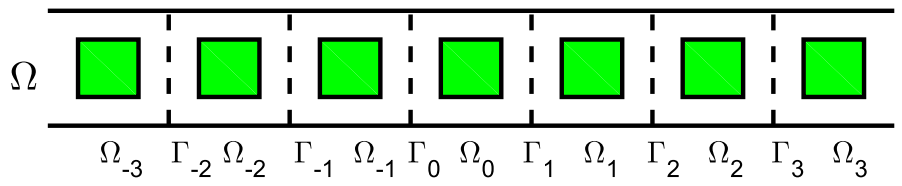

Fig. 1 Periodic waveguide

method to more general wavenumbers, with an interpolation technique inspired by the paper [5].

The numerical method is also extended to halfguide problems. From [20], any LAP solution of a halfguide problem is (not uniquely) extended to an LAP solution of a fullguide problem. Thus the numerical method can be designed as two steps. The first step is to find out the source term for the fullguide problems such that its solution approximates that of the halfguide problem, for given boundary data. We compute the boundary values for basis functions in the space where the source term lies in, and then find out the corresponding coefficients by the least square method that approximates the boundary data. As the choice of source terms is not unique and the problem is severely ill-posed, a Tikhonov regularization technique is adopted. With this source term, we go to the second step, i.e., to solve the fullguide problem with the method developed in this paper and approximate the solution of the halfguide problem. Moreover, the methods can also be extended to more general wavenumbers in the same way.

The rest of this paper is organized as follows. We recall the mathematical model of the scattering problem in the second section, and introduce the Floquet-Bloch theory in the third section. Then we consider the quasi-periodic cell problems in the fourth section. In Sect. 5, we apply the Floquet-Bloch transform to obtain a simplified integral representation of the LAP solutions. In Sect. 6, we develop a numerical method for LAP solutions, based on the integral representation. Then we extend the method to halfguide problems in Sect. 7 and more general wavenumbers in Sect. 8. In the last section, we present numerical results to show the efficiency of our algorithms.

\section{The mathematical model of direct scattering problems}

Let $\Omega=\mathbb{R} \times(0,1) \subset \mathbb{R}^{2}$ be a closed waveguide (see Fig. 1 ) with boundary

$$
\partial \Omega=\left\{x \in \mathbb{R}^{2}: x_{2}=0 \text { or } x_{2}=1\right\}
$$

The boundary is assumed to be impenetrable. Suppose $\Omega$ is filled up with periodic material with a real-valued refractive index $q$ satisfying:

$$
q\left(x_{1}+1, x_{2}\right)=q\left(x_{1}, x_{2}\right), \quad q \geq c_{0}>0 \quad \forall x \in \Omega .
$$

Moreover, we require that $q \in L^{\infty}(\Omega)$. 
The scattering problem is modeled by the following equations:

$$
\begin{aligned}
\Delta u+k^{2} q u & =f \quad \text { in } \Omega ; \\
\frac{\partial u}{\partial x_{2}} & =0 \quad \text { on } \partial \Omega,
\end{aligned}
$$

where $f$ is a function in $L^{2}(\Omega)$ with compact support, and $k>0$ is the real wavenumber.

Remark 1 We can also consider different boundary conditions on $\partial \Omega$, e.g., the Dirichlet boundary condition or the Robin boundary condition, in the similar way. In this paper, we only want to take the Neumann boundary condition as an example for our methods.

To find a "physically meaningful" solution of (1)-(2), we introduce the well-known Limiting Absorption Principle (LAP). That is, given any $\epsilon>0$, consider the following damped Helmholtz equation:

$$
\begin{aligned}
\Delta u_{\epsilon}+\left(k^{2}+\mathrm{i} \epsilon\right) q u_{\epsilon} & =f & & \text { in } \Omega ; \\
\frac{\partial u_{\epsilon}}{\partial x_{2}} & =0 & & \text { on } \partial \Omega .
\end{aligned}
$$

From the Lax-Milgram theorem, the problem is uniquely solvable in $H^{1}(\Omega)$. Moreover, the solution $u_{\epsilon}$ decays exponentially as $x_{1} \rightarrow \pm \infty$ (see [8]). The LAP assumes that $u_{\epsilon}$ converges in $H_{l o c}^{1}(\Omega)$ when $\epsilon \rightarrow 0^{+}$, and the limit, denoted by $u$, is the "physically meaningful" LAP solution. In the following, we introduce a new formulation for LAP solutions. Based on the new formulation, we introduce new numerical methods to compute the LAP solutions efficiently.

For simplicity, we introduce the following operator:

$$
\mathcal{A} u:=-q^{-1} \Delta u \text { with } D(\mathcal{A}, \Omega):=\left\{u \in H^{1}(\Omega): \Delta u \in L^{2}(\Omega),\left.\frac{\partial u}{\partial x_{2}}\right|_{\partial \Omega}=0\right\} \text {. }
$$

Let the spectrum of $\mathcal{A}$ be denoted by $\sigma(\mathcal{A})$, then the problem (1)-(2) is uniquely solvable in $H^{1}(\Omega)$ if and only if $k^{2} \notin \sigma(\mathcal{A})$. To study the spectrum property of $\mathcal{A}$ which plays an important role in the well-posedness of the problem (1)-(2), we introduce the Floquet-Bloch theory. For details we refer to [2,8] and for more general results we refer to [25]. 


\section{Floquet-Bloch theory and quasi-periodic problems}

\subsection{Floquet-Bloch theory}

In this section, we introduce the Floquet-Bloch theory to study the spectrum $\sigma(A)$. For simplicity, we introduce the following domains (see Fig. 1):

$$
\Omega_{j}:=(-1 / 2+j, 1 / 2+j] \times(0,1), \quad \Gamma_{j}=\{-1 / 2+j\} \times(0,1), \quad j \in \mathbb{Z} .
$$

Then $\Omega=\bigcup_{j \in \mathbb{Z}} \Omega_{j}$ with the left and right boundaries $\Gamma_{j}$ and $\Gamma_{j+1}$. Let

$$
\partial \Omega_{j}:=\partial \Omega \cap \overline{\Omega_{j}}=\left\{x \in \mathbb{R}^{2}:-1 / 2+j<x_{1} \leq 1 / 2+j, x_{2}=0 \text { or } 1\right\} .
$$

We also introduce the space of quasi-periodic functions. A function $\phi \in H_{l o c}^{1}(\Omega)$ is called $z$-quasi-periodic, if it satisfies

$$
\left.\phi\right|_{\Gamma_{j+1}}=\left.z \phi\right|_{\Gamma_{j}}, \quad \forall j \in \mathbb{Z}
$$

for some fixed complex number $z \in \mathbb{C}$. We define the subspace of $H^{1}\left(\Omega_{0}\right)$ by:

$$
H_{z}^{1}\left(\Omega_{0}\right):=\left\{\phi \in H^{1}\left(\Omega_{0}\right): \phi \text { satisfies (5) for } j=0\right\} .
$$

Then the functions in $H_{z}^{1}\left(\Omega_{0}\right)$ can be extended to $z$-quasi-periodic functions. Especially, when $z=1$, all functions in $H_{1}^{1}\left(\Omega_{0}\right)$ can be extended into periodic functions in $H_{l o c}^{1}(\Omega)$. We also denote $H_{1}^{1}\left(\Omega_{0}\right)$ by $H_{p e r}^{1}\left(\Omega_{0}\right)$.

From the Floquet-Bloch theory, the spectrum of $\mathcal{A}$ is closely related to Bloch wave solutions. A Bloch wave solution is a non-trivial solution of (1)-(2) in $H_{z}^{1}\left(\Omega_{0}\right)$ with $f=0$ for some $z \in \mathbb{C}$. If a Bloch wave solution exists in $H_{z}^{1}\left(\Omega_{0}\right), z$ is called a Floquet- multiplier. Define the operator:

$$
\mathcal{A}_{z} u=-q^{-1} \Delta u \text { with domain } D_{z}\left(\mathcal{A}, \Omega_{0}\right):=D\left(\mathcal{A}, \Omega_{0}\right) \cap H_{z}^{1}\left(\Omega_{0}\right),
$$

where $D\left(\mathcal{A}, \Omega_{0}\right)$ is defined in the same way as $D(\mathcal{A}, \Omega)$, and $\Omega$ is replaced by the periodic cell $\Omega_{0}$. Note that, $\mathcal{A}_{z}$ is self-adjoint with respect to the $L^{2}$-space equipped with the weighted inner product $(\phi, \psi)_{L^{2}, q}=\int_{\Omega_{0}} q \phi \bar{\psi} \mathrm{d} x$. Let $\sigma\left(\mathcal{A}_{z}\right)$ be the spectrum of $\mathcal{A}_{z}$, then $k^{2} \in \sigma\left(A_{z}\right)$ if and only if $z$ is a Floquet multiplier.

Let $\mathbb{F}\left(k^{2}\right)$ be the collection of all Floquet multipliers with wavenumber $k$ and $\mathbb{U F}\left(k^{2}\right):=\mathbb{F}\left(k^{2}\right) \cap \mathbb{S}^{1}\left(\mathbb{S}^{1}\right.$ is the unit circle in $\mathbb{C}$ ) be the set of all unit Floquet multipliers. In this paper, when the wavenumber is fixed, we write $\mathbb{F}$ instead of $\mathbb{F}\left(k^{2}\right)$ for simplicity. We list the properties of the Floquet multipliers [20], for more details we refer to $[2,6,8,21,25]$ :

- $\mathbb{U F}$ is finite.

$-z \in \mathbb{F}$ if and only if $z^{-1} \in \mathbb{F}$, thus $z \in \mathbb{U F}$ if and only if $\bar{z}=z^{-1} \in \mathbb{U} \mathbb{F}$.

$-\mathbb{F}$ is a discrete set and the only finite accumulation point of $\mathbb{F}$ can be 0 .

- $\mathbb{F}\left(k^{2}\right)$ depends continuously on $k^{2}$. 

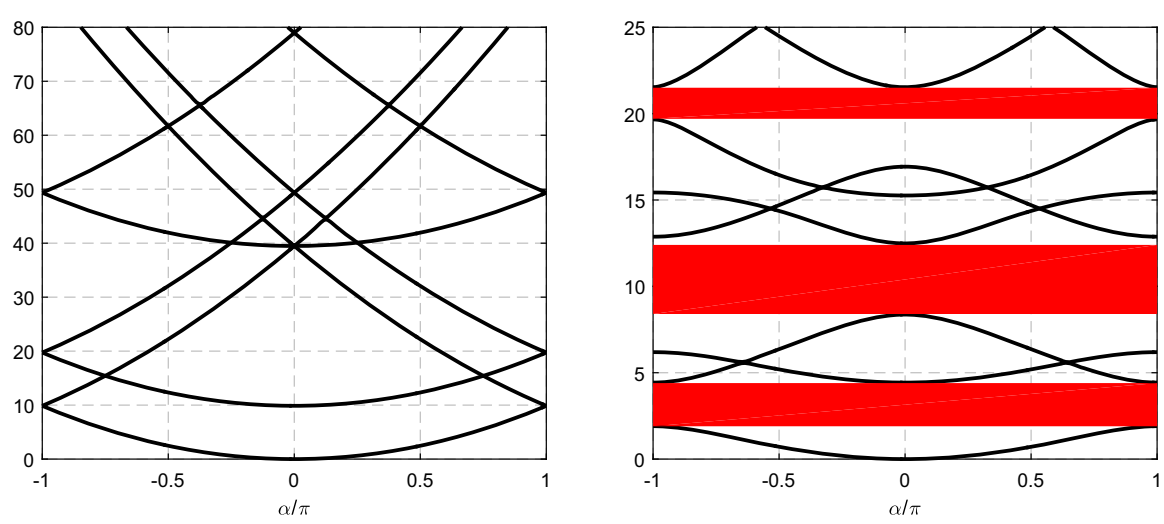

Fig. 2 Dispersion diagram. Left: Case I; Right: Case II

A classical result from the Floquet-Bloch theory also shows that (see [25]):

$$
\sigma(\mathcal{A})=\bigcup_{|z|=1} \sigma\left(\mathcal{A}_{z}\right)
$$

Thus, it is particularly important to study the spectrum of $\mathcal{A}_{z}$ when $|z|=1$. For simplicity, let $\alpha=-\mathrm{i} \log (z)$ where $\alpha \in(-\pi, \pi]$. We replace $\mathcal{A}_{z}$ by $\mathcal{A}_{\alpha}$ in the rest of this section, by abuse of notation. Then (5) becomes

$$
\left.u\right|_{\Gamma_{j+1}}=\left.\exp (\mathrm{i} \alpha) u\right|_{\Gamma_{j}}, \quad \forall j \in \mathbb{Z}
$$

Denote the spectrum of $\mathcal{A}_{\alpha}$ by $\sigma\left(\mathcal{A}_{\alpha}\right)$. As $\mathcal{A}_{\alpha}$ is self-adjoint, $\sigma\left(\mathcal{A}_{\alpha}\right)$ is a discrete subset of $(0, \infty)$. By rearranging the order of the points in $\sigma\left(\mathcal{A}_{\alpha}\right)$ properly, we obtain a family of analytic functions $\left\{\mu_{n}(\alpha): n \in \mathbb{N}\right\}$ and $\left\{\psi_{n}(\cdot, \alpha): n \in \mathbb{N}\right\}$ :

$$
\mathcal{A}_{\alpha} \psi_{n}(\cdot, \alpha)=\mu_{n}(\alpha) \psi_{n}(\cdot, \alpha), \quad \sigma\left(\mathcal{A}_{\alpha}\right)=\bigcup_{n \in \mathbb{N}}\left\{\mu_{n}(\alpha)\right\} .
$$

Thus $\sigma(\mathcal{A})=\bigcup_{n \in \mathbb{N}} \bigcup_{\alpha \in(-\pi, \pi]}\left\{\mu_{n}(\alpha)\right\}$. Both $\mu_{n}(\alpha)$ and $\psi_{n}(\cdot, \alpha)$ are extended into analytic functions in $\alpha$ in a sufficiently small neighbourhood of $(-\pi, \pi) \times\{0\}$.

For any fixed $n \in \mathbb{N}$, the graph $\left\{\left(\alpha, \mu_{n}(\alpha)\right): \alpha \in(-\pi, \pi]\right\}$ is called a dispersion curve, and all dispersion curves compose a dispersion diagram. Following [5,6], we first show the dispersion diagrams for two different examples:

1. Example 1. $q=1$ is a constant function, and its dispersion diagram is shown in Fig. 2 (left). The dispersion curve is given analytically:

$$
\mu_{j m}(\alpha)=j^{2} \pi^{2}+(\alpha+2 \pi m)^{2}, \quad j \in \mathbb{N}, m \in \mathbb{Z} .
$$

2. Example 2. $q=9$ in a disk $B((0,0.5), 0.3)$ and $q=1$ outside the disk, and its dispersion diagram is shown in Fig. 2 (right).. 
In the right picture of Fig. 2, there are "stop bands" (in red). When $k^{2}$ lies in the stop bands, the horizontal line with height $k^{2}$ has no intersection with any dispersion curves. This implies there is no propagating mode and the scattering problem (1)-(2) has a unique solution in $H^{1}(\Omega)$. The rest of bands are called "pass bands", such as the whole domain in the left picture of Fig. 2 and the white region in the right picture of Fig. 2. When $k^{2}$ lies in a pass band, from (7), there is at least one $\alpha \in(-\pi, \pi]$ such that there is a non-trivial $\alpha$-quasi-periodic function $\psi$ satisfying $\mathcal{A}_{\alpha} \psi=k^{2} \psi$. Thus it is a Bloch wave solution and is called a propagating Floquet mode. The case when $k^{2}$ lies in a pass band is particularly interesting and challenging. Thus we discuss more details about this case.

When $k^{2}$ lies in a pass band, there is at least one $\alpha \in(-\pi, \pi]$ such that $k^{2} \in \sigma\left(A_{\alpha}\right)$. Then the set

$$
P:=\left\{\alpha \in(-\pi, \pi]: \exists n \in \mathbb{N} \text {, s.t., } \mu_{n}(\alpha)=k^{2}\right\} \neq \emptyset .
$$

Thus $\mathbb{U F}$ can be written as:

$$
\mathbb{U F}=\{\exp (\mathrm{i} \alpha): \alpha \in P\}
$$

The points in $P$ are divided into the following three classes:

- When $\mu_{n}^{\prime}(\alpha)>0, \psi_{n}(\cdot, \alpha)$ propagates from the left to the right;

- when $\mu_{n}^{\prime}(\alpha)<0, \psi_{n}(\cdot, \alpha)$ propagates from the right to the left;

- when $\mu_{n}^{\prime}(\alpha)=0$, we can not decide the direction that $\psi_{n}(\cdot, \alpha)$ propagates.

For physical interpretations of the Floquet modes $\psi_{n}(\cdot, \alpha)$ we refer to Remark 4, [2]. Based on the above classification, we define the following three sets:

$$
\begin{aligned}
& P_{ \pm}:=\left\{\alpha \in(-\pi, \pi]: \exists n \in \mathbb{N} \text { s.t., } \mu_{n}(\alpha)=k^{2} \text { and } \pm \mu_{n}^{\prime}(\alpha)>0\right\} \\
& P_{0}:=\left\{\alpha \in(-\pi, \pi]: \exists n \in \mathbb{N} \text { s.t., } \mu_{n}(\alpha)=k^{2} \text { and } \mu_{n}^{\prime}(\alpha)=0\right\}
\end{aligned}
$$

Then $P=P_{+} \bigcup P_{-} \bigcup P_{0}$.

Remark 2 It is possible that there are two (or more) different dispersion curves passing through the point $\left(\alpha, k^{2}\right)$. Suppose the elements in $P$ have $Q$ different values $\alpha_{1}, \ldots, \alpha_{Q}$. For any $j=1,2, \ldots, Q$, there are $L_{j}\left(L_{j} \geq 1\right)$ different dispersion curves $\mu_{j, \ell}$ passing through $\left(\alpha, k^{2}\right)\left(\ell=1,2, \ldots, L_{j}\right)$ such that

$$
\mu_{j, 1}\left(\alpha_{j}\right)=\mu_{j, 2}\left(\alpha_{j}\right)=\cdots \mu_{j, L_{j}}\left(\alpha_{j}\right)=k^{2} .
$$

In this case, $\alpha_{j}$ is treated as $L_{j}$ different elements in $P$, i.e.,

$$
\alpha_{j, 1}=\alpha_{j, 2}=\cdots=\alpha_{j, L_{j}}=\alpha_{j}
$$

As $\mathbb{U F}$ is symmetric, $P_{ \pm}$is also symmetric, i.e., $\alpha \in P_{+}$if and only if $-\alpha \in P_{-}$. For details see Theorem 4, [2]. 

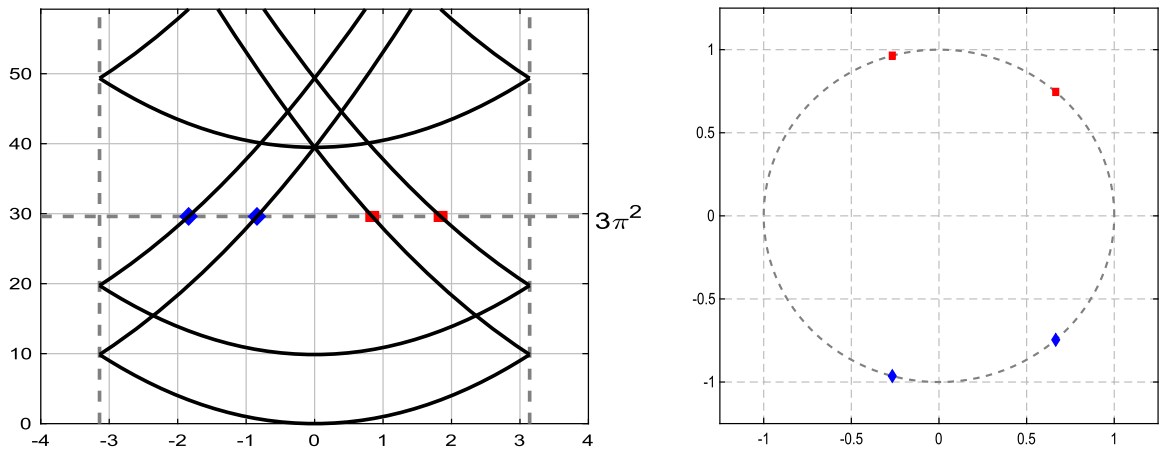

Fig. 3 Example for $n=1$ and $k^{2}=3 \pi^{2}$. UF in $\alpha$-space and $z$-space. Red squares denotes the points in $P_{-}\left(\S_{-}^{0}\right)$, while blue diamonds denotes the points in $P_{+}\left(\S_{+}^{0}\right)$

As the limiting absorption principle fails when the set $P_{0}$ is not empty, we make the following assumption.

Assumption 1 Assume that in this paper, $P_{0}=\emptyset$.

The assumption is reasonable as the set $\left\{k>0: P_{0} \neq \emptyset\right\}$ is "sufficiently small", i.e., the set is countable with at most one accumulation point at $\infty$ (see Theorem 5, [2]).

In our later proof of the new integral representation of LAP solutions, we also have to avoid the cases when $P_{+} \cap P_{-} \neq \emptyset$. Luckily, with the similar method used in the proof of Theorem 5 in [2], we can also prove that this set is discrete in the following lemma. For the proof we refer to Appendix.

Lemma 1 The set $\left\{k \in \mathbb{R}_{+}: P_{+} \cap P_{-} \neq \emptyset\right\}$ is countable, and its only accumulation point is $\infty$.

Assumption 2 Until Sect. 7, we assume that $k$ satisfies $P_{+} \cap P_{-}=\emptyset$.

We define three subsets of $\mathbb{U F}$ from the definition of $P_{ \pm}$and $P_{0}$ by:

$$
S_{ \pm}^{0}:=\left\{z=\exp (\mathrm{i} \alpha): \alpha \in P_{ \pm}\right\}, \quad S_{0}^{0}:=\left\{z=\exp (\mathrm{i} \alpha): \alpha \in P_{0}\right\}
$$

Then $\mathbb{U F}=S_{+}^{0} \bigcup S_{-}^{0} \bigcup S_{0}^{0}$.

We also divide the set $\mathbb{F} \backslash \mathbb{U F}$ into the following two subsets:

$$
R S:=\{z \in \mathbb{F}:|z|<1\} ; \quad L S:=\{z \in \mathbb{F}:|z|>1\}
$$

The Bloch wave solution corresponding to $z \in R S$ is evanescent on the right, while the one corresponding to $z \in L S$ is evanescent on the left. Moreover, let

$$
S_{+}:=S_{+}^{0} \bigcup R S, \quad S_{-}:=S_{-}^{0} \bigcup L S .
$$

Remark 3 We conclude the properties of the sets $R S, L S$ and $S_{ \pm}^{0}$ from the properties of $\mathbb{F}$ as follows: 
- From the symmetry of $\mathbb{F}$, the sets $S_{+}^{0}$ and $S_{-}^{0}, R S$ and $L S$ are symmetric, i.e.,

$$
z \in S_{+}^{0} \Longleftrightarrow z^{-1}=\bar{z} \in S_{-}^{0} ; \quad z \in R S \Longleftrightarrow z^{-1} \in L S .
$$

This also implies that

$$
z \in S_{+} \Longleftrightarrow z^{-1} \in S_{-} .
$$

- When Assumption 1 is satisfied, $S_{0}^{0}=\emptyset$, thus $S_{+}^{0} \cup S_{-}^{0}=\mathbb{U F}$.

- When Assumption 2 is satisfied, $S_{+}^{0} \cap S_{-}^{0}=\emptyset$.

- From (10), if $\pm 1 \in S_{+}^{0}$ (or $\pm 1 \in S_{-}^{0}$ ), then $\pm 1 \in S_{-}^{0} \cap S_{+}^{0}$. If Assumption 2 is satisfied, $S_{+}^{0} \cap S_{-}^{0}=\emptyset$, then $\pm 1 \notin \mathbb{U F} \backslash S_{0}^{0}$. If Assumption 1 is also satisfied, $S_{0}^{0}=\emptyset$ implies that $\pm 1 \notin \mathbb{F}$.

Lemma 2 Let $k>0$. There is a $\tau>0$ such that $R S \in B(0, \exp (-\tau))$ and $L S \in$ $\mathbb{C} \backslash \overline{B(0, \exp (\tau))}$.

We omit the proof here. For details we refer to [20].

\subsection{Quasi-periodic problems}

From the last section, quasi-periodic problems are very important in the investigation of scattering problems in periodic domains. In this section, we consider the $z$-dependent cell problem:

$$
\begin{aligned}
\Delta u_{z}+k^{2} q u_{z} & =f_{z} \quad \text { in } \Omega_{0} ; \\
\frac{\partial u_{z}}{\partial x_{2}} & =0 \quad \text { on } \partial \Omega \cap \overline{\Omega_{0}} ; \\
\left.u\right|_{\Gamma_{1}}-\left.z u\right|_{\Gamma_{0}} & =0 ;
\end{aligned}
$$

where $z \neq 0$ is a complex number and $f_{z} \in L^{2}\left(\Omega_{0}\right)$ depends analytically on $z$.

We are interested in the well-posedness of the problem (11)-(13), and also the dependence of the solution $u_{z}$ on the quasi-periodicity $z$. To this end, it is more convenient to study the problems in a fixed domain. Let $v_{z}=z^{-x_{1}} u_{z}(x)$, then $v_{z} \in H_{p e r}^{1}\left(\Omega_{0}\right)$. Note that as $z^{-x_{1}}$ is a multi-valued function, we require that $z$ lies in the branch cutting off along the negative real axis (denoted by $\mathbb{C}_{\times}:=\{z \in \mathbb{C} \backslash\{0\}$ : $-\pi<\arg (z) \leq \pi\}$, where $\arg (z)$ is the argument of the complex number $z$ ). From direct calculation, $v_{z}$ is the solution of the following problem:

$$
\begin{aligned}
\Delta v_{z}+2 \log (z) \frac{\partial v_{z}}{\partial x_{1}}+\left(k^{2} q+\log ^{2}(z)\right) v_{z} & =z^{-x_{1}} f_{z} \quad \text { in } \Omega_{0} ; \\
\frac{\partial v_{z}}{\partial v} & =0 \quad \text { on } \partial \Omega_{0} ; \\
\left.v_{z}\right|_{\Gamma_{1}}-\left.v_{z}\right|_{\Gamma_{0}} & =0 .
\end{aligned}
$$


For each fixed $z$, the problem is equivalent to the following equation. For definitions of the corresponding operators/elements we refer to Sect. 4, [20].

$$
\left(I-\mathcal{K}_{z}\right) v_{z}=z^{-x_{1}} \tilde{f}_{z}
$$

This implies that when $I-\mathcal{K}_{z}$ is invertible, then

$$
v_{z}=\left(I-\mathcal{K}_{z}\right)^{-1} z^{-x_{1}} \widetilde{f_{z}} .
$$

As $\mathcal{K}_{z}$ is compact and depends analytically on $z \in \mathbb{C}_{\times}, I-\mathcal{K}_{z}$ is an analytic family of Fredholm operators. From the Analytic Fredholm Theory (see Theorem VI.14, [26]), $\left(I-\mathcal{K}_{z}\right)^{-1}$ depends meromorphically on $z$ in $\left.\mathbb{C} \backslash\{0\}\right\}$. From Sect. 4 in [20], the set of poles of $\left(I-\mathcal{K}_{z}\right)^{-1}$ is exactly $\mathbb{F}$. Thus $v_{z}$, or equivalently $u_{z}=z^{x_{1}} v(x)$, depends analytically on $z \in \mathbb{C} \backslash(\mathbb{F} \bigcup\{0\})$ and meromorphically on $z \in \mathbb{C} \backslash\{0\}$.

\section{The Floquet-Bloch transform and its application}

\subsection{The Floquet-Bloch transform}

The Floquet-Bloch transform is a very important tool in the analysis of scattering problems in PDEs in periodic structures, see [2-4].

For a function $\phi \in C_{0}^{\infty}(\Omega)$, define the Floquet-Bloch transform of $\phi$ by

$$
(\mathcal{F} \phi)(z, x):=\sum_{n=-\infty}^{\infty} \phi\left(x_{1}+n, x_{2}\right) z^{-n}, \quad x \in \Omega_{0}, z \in \mathbb{C} .
$$

The transform is well-defined for any smooth function with compact support, and it can be extended to more general cases. Define the Region of Convergence (ROC) as the domain in $\mathbb{C}$ such that the series (15) converges. Note that the DOC may be empty for given function $\phi$. When $\phi$ decays exponentially at the rate $\gamma$, i.e., there is a $\gamma>0$ and $C>0$ such that $\phi$ satisfies

$$
\left|\phi\left(x_{1}, x_{2}\right)\right| \leq C \exp \left(-\gamma\left|x_{1}\right|\right), \quad \forall x \in \Omega
$$

the Floquet-Bloch transform of $\phi$ is still well-defined, and the ROC is the annulus

$$
T_{\gamma}=\{z \in \mathbb{C}: \exp (-\gamma)<|z|<\exp (\gamma)\}
$$

Moreover, from the perturbation theory, the function $(\mathcal{F} \phi)(z, \cdot)$ depends analytically on $z \in T_{\gamma}$. It is also easy to check that the transformed function $(\mathcal{F} \phi)(z, \cdot)$ is quasiperiodic (i.e., it satisfies (5)). We conclude some mapping properties of the operator $\mathcal{F}$ in the following proposition.

Proposition 1 The operator $\mathcal{F}$ has the following properties when $z$ lies on the unit circle $\mathbb{S}^{1}$ (see $\left.[11,21]\right)$ : 
- $\mathcal{F}$ is an isomorphism between $H^{s}(\Omega)$ and $L^{2}\left(\mathbb{S}^{1} ; H_{z}^{s}\left(\Omega_{0}\right)\right)(s \in \mathbb{R})$, where

$$
L^{2}\left(\mathbb{S}^{1} ; H_{z}^{s}\left(\Omega_{0}\right)\right):=\left\{\phi \in \mathcal{D}^{\prime}\left(\mathbb{S}^{1} \times \Omega_{0}\right):\left[\int_{\mathbb{S}^{1}}\|\phi(z, \cdot)\|_{H_{z}^{s}\left(\Omega_{0}\right)}^{2} \mathrm{~d} z\right]^{1 / 2}<\infty\right\}
$$

- $\mathcal{F} \phi$ depends analytically on $z \in T_{\gamma}$, if and only if $\phi$ decays exponentially with the rate $\gamma$.

- Given $\psi(z, x):=(\mathcal{F} \phi)(z, x)$ for some $\phi \in H^{s}(\Omega)$ and satisfies (16) for some $\gamma>0$, the inverse operator $\mathcal{F}$ is given by:

$$
\left(\mathcal{F}^{-1} \psi\right)\left(x_{1}+n, x_{2}\right)=\frac{1}{2 \pi \mathrm{i}} \oint_{\mathbb{S}^{1}} \psi(z, x) z^{n-1} \mathrm{~d} z .
$$

\subsection{Application of the Floquet-Bloch transform}

In this section, we apply the Floquet-Bloch transform $\mathcal{F}$ to the scattering problem (1)-(2), when $k^{2} \notin \sigma(A)$. We are particularly interested in the case:

- for $k>0, k^{2} \notin \sigma(A)$, i.e., $k^{2}$ lies in a stop band; or

$-k^{2}$ is no longer real, i.e., $k^{2}=k_{0}^{2}+\mathrm{i} \epsilon$ for some fixed $k_{0}>0$ and $\epsilon>0$.

When either of the two conditions is satisfied, the problem (1)-(2) is uniquely solvable in $H^{1}(\Omega)$. Moreover, $u$ decays exponentially at the infinity, i.e., $u$ satisfies (16) for some $C>0$ and $\gamma>0$ (see [24]).

Remark 4 From now on, we assume that $\operatorname{supp}(f) \subset \Omega_{0}$. The results are easily extended to cases when $\operatorname{supp}(f)$ lies in larger bounded domains.

We define the Floquet-Bloch transform $w(z, x):=(\mathcal{F} u)(z, x)$, then the transformed field $w(z, \cdot)$ is well-defined and depends analytically on $z \in T_{\gamma}$ as a $H^{1}\left(\Omega_{0}\right)$-function valued function. It is also easy to check that for any $z \in T_{\gamma}$, $w(z, \cdot) \in H_{z}^{1}\left(\Omega_{0}\right)$ satisfies (11)-(13). Note that the source term in (11) is $f$, as $(\mathcal{F} f)(z, x)=f(x)$ for any $z \in T_{\gamma}$.

From the inverse Floquet-Bloch transform and Cauchy integral theorem, the solution of the original problem can be represented as:

$$
\begin{aligned}
u\left(x_{1}+n, x_{2}\right)=\left(\mathcal{F}^{-1} w\right)\left(x_{1}+n, x_{2}\right) & =\frac{1}{2 \pi \mathrm{i}} \oint_{\mathbb{S}^{1}} w(z, x) z^{n-1} \mathrm{~d} z \\
& =\frac{1}{2 \pi \mathrm{i}} \oint_{\mathcal{C}} w(z, x) z^{n-1} \mathrm{~d} z,
\end{aligned}
$$

where $\mathcal{C}$ is a rectifiable curve in $T_{\gamma}$ encircling 0 .

From the exponential decay of $u,(\mathcal{F} u)(z, \cdot)$ exists and depends analytically on $z \in T_{\gamma}$. On the other hand, from Sect. 3.2, when $z \in \mathbb{C} \backslash \mathbb{F}$, the problem (11)-(13) is uniquely solvable in $H_{z}^{1}\left(\Omega_{0}\right)$, and $w(z, \cdot)$ depends analytically on $z \in \mathbb{C} \backslash(\mathbb{F} \bigcup\{0\})$ and meromorphically on $z \in \mathbb{C} \backslash\{0\}$. Thus $(\mathcal{F} u)(z, \cdot)$ is extended meromorphically in $\mathbb{C} \backslash\{0\}$. From the analytic continuation, we obtain the following result (see [20]). 
Theorem 1 When $k^{2} \notin \sigma(\mathcal{A})$, the Floquet-Bloch transformed field $(\mathcal{F} u)(z, x)$ is extended to an analytic function in $\mathbb{C} \backslash(\mathbb{F} \cup\{0\})$ and a meromorphic function in $\mathbb{C} \backslash\{0\}$ by the solution $w(z, \cdot)$ of $(11)-(13)$.

The integral representation of $u$ is obtained from Cauchy integral theorem.

Theorem 2 Suppose $k^{2} \notin \sigma(\mathcal{A})$. $w(z, \cdot)$ is the solution of (11)-(13) for $z \in \mathbb{C} \backslash \mathbb{F}$. Then the solution of (1)-(2) is written as

$$
u\left(x_{1}+n, x_{2}\right)=\left(\mathcal{F}^{-1} w\right)\left(x_{1}+n, x_{2}\right)=\frac{1}{2 \pi i} \oint_{\mathcal{C}} w(z, x) z^{n-1} \mathrm{~d} z,
$$

where $\mathcal{C} \subset \mathbb{C}$ is a counter-clockwise closed rectifiable path encircling all the points in $R S\left(=S_{+}\right)$and does not encircle any point in $L S\left(=S_{-}\right)$.

\section{The Limiting absorption principle (LAP)}

In this section, we consider the case when $k^{2} \in \sigma(\mathcal{A})$ with the help of the limiting absorption principle. In the first two sections, we always assume that Assumption 2 holds. First we consider the damped Helmholtz equation (3)-(4). The corresponding $z$-quasi-periodic problem is formulated as:

$$
\begin{aligned}
\Delta w_{\epsilon}(z, \cdot)+\left(k^{2}+\mathrm{i} \epsilon\right) q w_{\epsilon}(z, \cdot) & =f \quad \text { in } \Omega_{0} ; \\
\frac{\partial w_{\epsilon}(z, \cdot)}{\partial x_{2}} & =0 \quad \text { on } \partial \Omega_{0} ; \\
\left.w_{\epsilon}(z, \cdot)\right|_{\Gamma_{1}}-\left.z w_{\epsilon}(z, \cdot)\right|_{\Gamma_{0}} & =0 .
\end{aligned}
$$

Similar to the definition of $\mathcal{K}_{z}$, we denote the operator with $k^{2}+\mathrm{i} \epsilon$ by $\mathcal{K}_{z}^{\epsilon}$. From Theorem 4 in [22], the poles of the operator $\left(I-\mathcal{K}_{z}^{\epsilon}\right)^{-1}$ depend continuously on $\epsilon$. First, we study the asymptotic behaviour of distribution of the poles when $\epsilon>0$ is sufficiently small.

\subsection{Distribution of poles of the damped Helmholtz equations}

From the Floquet-Bloch theory (see $[2,25]), k^{2} \in \sigma(\mathcal{A})$ implies that $\mathbb{U} \neq \varnothing$. For any $z \in \mathbb{U F}, k^{2}$ is an eigenvalue of $\mathcal{A}_{z}$. As both $S_{+}^{0}$ and $S_{-}^{0}$ are finite sets, and $S_{+}^{0}$ and $S_{-}^{0}$, $R S$ and $L S$ are symmetric in the sense of (10), they are written as

$$
\begin{aligned}
& S_{+}^{0}=\left\{z_{j, \ell}^{+}: j=1, \ldots, Q ; \ell=1, \ldots, L_{j}\right\} ; R S=\left\{z_{Q+1}^{+}, z_{Q+2}^{+}, \ldots\right\} \\
& S_{-}^{0}=\left\{z_{j, \ell}^{-}: j=1, \ldots, Q ; \ell=1, \ldots, L_{j}\right\} ; \quad L S=\left\{z_{Q+1}^{-}, z_{Q+2}^{-}, \ldots\right\}
\end{aligned}
$$

where $z_{j}^{+}(j=1, \ldots, Q)$ are $Q$ different values on the unit circle, and so are $z_{j}^{-}$ $(j=1, \ldots, Q)$. Moreover, $z_{j, 1}^{ \pm}=\cdots=z_{j, L_{j}}^{ \pm}=z_{j}^{ \pm}$for $j=1, \ldots, Q$, and $z_{j}^{+}=$ 
$\left(z_{j}^{-}\right)^{-1}$ for any integer $j \in \mathbb{N}$. From Assumption 1 and $2, \mathbb{U F}=S_{+}^{0} \cup S_{-}^{0}, \mathbb{F}=$ $S_{+}^{0} \cup S_{-}^{0} \cup R S \bigcup L S$; moreover, $S_{+}^{0} \cap S_{-}^{0}=\emptyset$.

From the continuous dependence of poles, for any $z_{j, \ell}^{ \pm}$or $z_{j}^{ \pm} \in \mathbb{F}$ with $j \in \mathbb{N}$, there is a continuous function $Z_{j, \ell}^{ \pm}(\epsilon)$ or $Z_{j}^{ \pm}(\epsilon)$, such that $\left\{Z_{j, \ell}^{+}(\epsilon), Z_{j, \ell}^{-}(\epsilon): j=\right.$ $\left.1, \ldots, Q ; \ell=1, \ldots, L_{j}\right\} \bigcup\left\{Z_{j}^{+}(\epsilon), Z_{j}^{-}(\epsilon): j \geq Q+1\right\}$ are exactly the set of all poles with respect to $k^{2}+\mathrm{i} \epsilon$. Moreover, $\lim _{\epsilon \rightarrow 0} Z_{j, \ell}^{ \pm}(\epsilon)=Z_{j, \ell}^{ \pm}(0)=z_{j}^{ \pm}$for $j=1, \ldots, Q$ and $\ell=1, \ldots, L_{j}$ and $\lim _{\epsilon \rightarrow 0} Z_{j}^{ \pm}(\epsilon)=Z_{j}^{ \pm}(0)=z_{j}^{ \pm}$for $j \geq Q+1$. Analogous to the case $\epsilon=0$, we define the following sets depending on $\epsilon$ :

$$
\begin{aligned}
& S_{+}^{0}(\epsilon)=\left\{Z_{j, \ell}^{+}(\epsilon): j=1, \ldots, Q ; \ell=1, \ldots, L_{j}\right\} ; \\
& S_{-}^{0}(\epsilon)=\left\{Z_{j, \ell}^{-}(\epsilon): j=1, \ldots, Q ; \ell=1, \ldots, L_{j}\right\} ; \\
& L S(\epsilon)=\left\{Z_{Q+1}^{-}(\epsilon), Z_{Q+2}^{-}(\epsilon), \ldots\right\} ; \\
& R S(\epsilon)=\left\{Z_{Q+1}^{+}(\epsilon), Z_{Q+2}^{+}(\epsilon), \ldots\right\} .
\end{aligned}
$$

From the continuous dependence of $\epsilon>0$, we have the following properties of $Z_{j}^{ \pm}(\epsilon)$ when $j=1,2, \ldots, Q$. For the proof we refer to Appendix in [8].

Lemma 3 For any $j=1,2, \ldots, Q$, when $\epsilon>0$ is sufficiently small, the functions satisfy $\left|Z_{j, \ell}^{+}(\epsilon)\right|<1$ and $\left|Z_{j, \ell}^{-}(\epsilon)\right|>1$.

Thus we conclude the behaviour of $Z_{j}^{ \pm}(\epsilon)$ for sufficiently small $\epsilon$ :

- for any $z_{j}^{+} \in S_{+}^{0}$, the points $Z_{j, \ell}^{+}(\epsilon) \in S_{+}^{0}(\epsilon)$ converge to $z_{j}^{+}$from the interior of the unit circle;

- for any $z_{j}^{-} \in S_{-}^{0}$, the points $Z_{j, \ell}^{-}(\epsilon) \in S_{-}^{0}(\epsilon)$ converge to $z_{j}^{-}$from the exterior of the unit circle.

To make it clear, we present a visualization of examples of the curves in Fig. 4. The red rectangles are points in $S_{-}^{0}$ and the blue diamonds are points in $S_{+}^{0}$. The asymptotic behavior of $Z_{j, \ell}^{ \pm}(\epsilon)$ as $\epsilon \rightarrow 0$ can be seen from the picture.

For the points in $R S(\epsilon)$ or $L S(\epsilon)$, we estimate their distributions for sufficiently small $\epsilon>0$ in the following lemma (see Lemma 17, [20]).

Lemma 4 Supposefor some $\tau>0, R S \subset B(0, \exp (-\tau))$ and $L S \subset \mathbb{C} \backslash \overline{B(0, \exp (\tau))}$. For any $\tau_{1} \in(0, \tau)$, there exists $\epsilon_{0}>0$ such that $Z_{j}^{+}(\epsilon) \in B\left(0, \exp \left(-\tau_{1}\right)\right)$ and $Z_{j}^{-}(\epsilon) \in \mathbb{C} \backslash \overline{B\left(0, \exp \left(\tau_{1}\right)\right)}$ for any $j \geq Q+1$ and $\epsilon \in\left(0, \epsilon_{0}\right)$.

From Lemmas 3 and 4 , when $\epsilon>0$ is sufficiently small, the sets $S_{ \pm}^{0}(\epsilon)$ and $R S(\epsilon)$, $L S(\epsilon)$ have following properties:

$$
S_{+}^{0}(\epsilon) \bigcup R S(\epsilon) \subset B(0,1) ; \quad S_{-}^{0}(\epsilon) \bigcup L S(\epsilon) \subset \mathbb{C} \backslash \overline{B(0,1)} .
$$



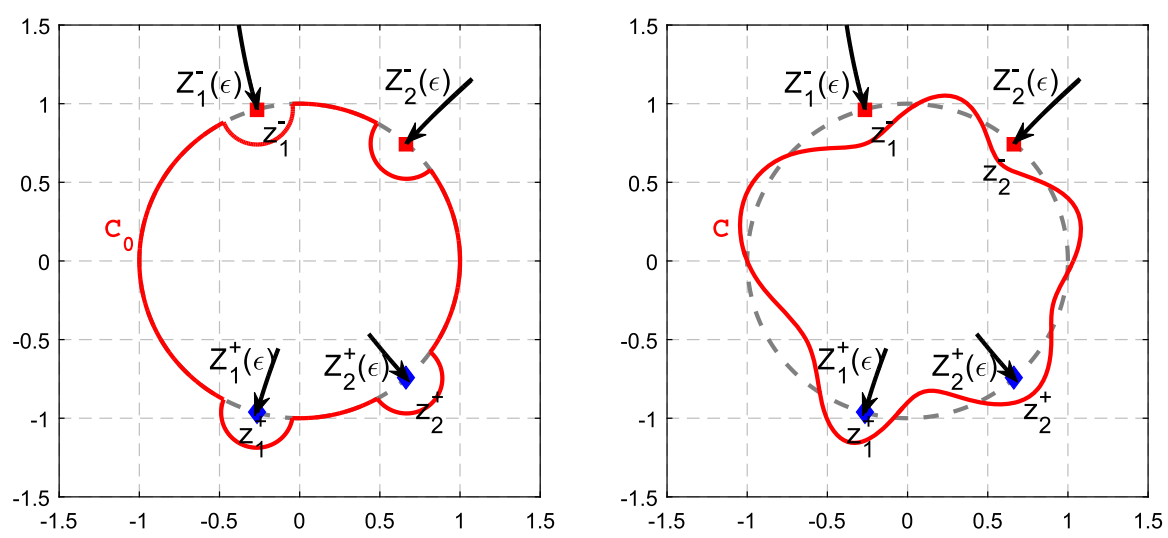

Fig. 4 Left: the curve $\mathcal{C}_{0}$; Right: a choice of $\mathcal{C}$. Red solid curves: $Z_{j}^{ \pm}(\epsilon)$; grey dashed curves: the unit circle.

The red rectangles are points in $S_{-}^{0}$ and the blue diamonds are points in $S_{+}^{0}$. The arrows show the direction of the poles when $\epsilon \rightarrow 0$

\subsection{Integral representation of the LAP solution}

Now we are prepared to formulate the LAP solution of (1)-(2) when $k^{2} \in \sigma(\mathcal{A})$. From Theorem 2, the solution $u_{\epsilon}(\forall \epsilon>0)$ of the damped problem (3)-(4) is represented by the curve integral:

$$
u_{\epsilon}\left(x_{1}+n, x_{2}\right)=\frac{1}{2 \pi \mathrm{i}} \oint_{\mathbb{S}^{1}} w_{\epsilon}(z, x) z^{n-1} \mathrm{~d} z
$$

But when $\epsilon \rightarrow 0^{+}$, as the poles in $S_{ \pm}^{0}(\epsilon)$ approach $\mathbb{S}^{1}$, and the integral becomes singular. From Theorem 2, we are aimed at finding out a proper curve $\mathcal{C}$ to replace the unit circle such that the function $w_{\epsilon}(z, x)$ is well-posed and converges uniformly with respect to $z$ when $\epsilon \rightarrow 0$. From the properties of the sets $S_{ \pm}^{0}(\epsilon), R S(\epsilon)$ and $L S(\epsilon)$, we define a convenient curve $\mathcal{C}_{0}$ as follows.

Definition 1 Let the piecewise analytic curve $\mathcal{C}_{0}$ be defined as the boundary of the following domain :

$$
B_{\delta}:=B(0,1) \bigcup\left[\bigcup_{n=1}^{Q} B\left(z_{j}^{+}, \delta\right)\right] \backslash\left[\bigcup_{n=1}^{Q} \overline{B\left(z_{j}^{-}, \delta\right)}\right]
$$

where $\delta>0$ is sufficiently small such that the following conditions are satisfied:

- For any $j \neq \ell, B\left(z_{j}^{ \pm}, \delta\right) \cap B\left(z_{\ell}^{ \pm}, \delta\right)=\emptyset$.

$-\left[\bigcup_{n=1}^{Q} B\left(z_{j}^{+}, \delta\right)\right] \cup\left[\bigcup_{n=1}^{Q} B\left(z_{j}^{-}, \delta\right)\right] \subset T_{\tau}$, i.e., the balls do not contain any point in $R S \cup L S$. 
From Assumption 2 and Lemma 2, we can always find a proper parameter $\delta$ such that both conditions are satisfied. We refer to Fig. 4 for a visualization of $\mathcal{C}_{0}$ for the example when $n=1$ and $k^{2}=3 \pi^{2}$.

Thus from Lemmas 3 and 4, there is a constant $C=C(\delta)>0$ such that

$$
d\left(\mathcal{C}_{0}, R S(\epsilon) \bigcup L S(\epsilon) \bigcup S_{+}^{0}(\epsilon) \bigcup S_{-}^{0}(\epsilon)\right)>C(\delta)
$$

holds uniformly for any fixed sufficiently small $\epsilon>0$, where $d(X, Y)$ is the Hausdorff distance between two subsets $X, Y \subset \mathbb{C}$. From the choice of the curve $\mathcal{C}_{0}$, the interior of the symmetric difference of $B_{\delta}$ and $B(0,1)$ is

$$
\left[\bigcup_{n=1}^{Q}\left(B\left(z_{n}^{+}, \delta\right) \backslash \overline{B(0,1)}\right)\right] \bigcup\left[\bigcup_{n=1}^{Q}\left(B\left(z_{n}^{-}, \delta\right) \cap B(0,1)\right)\right]
$$

As for sufficiently small $\epsilon>0,\left(I-\mathcal{K}_{z}^{\epsilon}\right)^{-1}$ depends analytically on $z$ in the above domain, from Cauchy integral theorem, $u_{\epsilon}$ has the equivalent formulation

$$
u_{\epsilon}\left(x_{1}+n, x_{2}\right)=\frac{1}{2 \pi \mathrm{i}} \oint_{\mathcal{C}_{0}} w_{\epsilon}(z, x) z^{n-1} \mathrm{~d} z .
$$

From the asymptotic behaviours of the poles $Z_{j}^{ \pm}(\epsilon)$ explained in Lemmas 3-4, let $\epsilon$ tends to $0^{+}$, we get the exact formulation for the LAP solution:

$$
u\left(x_{1}+n, x_{2}\right)=\frac{1}{2 \pi \mathrm{i}} \oint_{\mathcal{C}_{0}} w(z, x) z^{n-1} \mathrm{~d} z
$$

We also refer to Sect. 6.2, [20] for a similar approach. Moreover, for any $n \in \mathbb{Z}$, there is a constant $C=C(n)>0$ such that

$$
\|u\|_{H^{1}\left(\Omega_{n}\right)} \leq C\|f\|_{L^{2}\left(\Omega_{0}\right)}
$$

Moreover, from regularity of elliptic equations, the LAP solution $u \in H_{l o c}^{2}(\Omega)$.

We can also replace $\mathcal{C}_{0}$ by any closed curve that lies in the neighbourhood of $\mathcal{C}_{0}$ and enclose zero and all poles in $S_{+}$, but no poles in $S_{-}$(see Fig. 4 (right)). The left curve is $\mathcal{C}_{0}$, and the right is a choice of $\mathcal{C}$. Thus

$$
u\left(x_{1}+n, x_{2}\right)=\frac{1}{2 \pi \mathrm{i}} \oint_{\mathcal{C}} w(z, x) z^{n-1} \mathrm{~d} z
$$

Now we have already formulated the LAP solution directly from cell problems (19)-(21), which also provides a nice and clear formulation for the numerical scheme. However, we still need to know the dispersion diagram to find the correct curve $\mathcal{C}_{0}$ (or C). 


\subsection{Formulation for more general case}

The formulation (25) is based on Assumption 2, which is not necessary for the LAP process. In this section, we recall another formulation introduced in [20] which does not require it.

For fixed $k>0$, we recall the sets $P, P_{ \pm}$and $P_{0}$ (also the corresponding $S, S_{ \pm}$ and $S_{0}$ ). With Assumption $1, P_{0}=\emptyset$ thus $P=P_{+} \cup P_{-}$(and also $S=S_{+} \cup S_{-}$). However, since $P_{+} \cap P_{-}$may not be empty, we can not use the old notations any more. Instead, we simply let $P=\left\{\beta_{1}, \beta_{2}, \ldots, \beta_{Q}\right\}$ and each $\beta_{j}(j=1,2, \ldots, Q)$ is related to $L_{j}$ analytic functions $\mu_{j, \ell}\left(\ell=1,2, \ldots, L_{J}\right)$ which define $L_{j}$ dispersion diagrams. Thus if $\mu_{j, \ell}^{\prime}\left(\beta_{j}\right)>0(<0)$ then $\beta_{j} \in P_{+}\left(P_{-}\right)$.

Let $\psi_{j, \ell}(\beta, \cdot)$ be the eigenfunctions corresponding to the eigenvalues $\mu_{j, \ell}(\beta)$. We choose a $\tau>0$ such that the circle $|z|=\exp (-\tau)$ enclose all poles in $R S$, then from [20] with a similar complex contour integral with the LAP process, for all $n \in \mathbb{Z}$ :

$$
u\left(x_{1}+n, x_{2}\right)= \begin{cases}\mathrm{i}\left[\sum_{j=1}^{Q} \sum_{\left(\mu_{j, \ell}^{\prime}\left(\beta_{j}\right)>0\right.} \frac{\exp \left(\mathrm{in} \beta_{j}\right)\left\langle f, \psi_{j, \ell}\left(\beta_{j}, \cdot\right)\right\rangle}{\left(\mu_{j, \ell}\right)^{\prime}\left(\beta_{j}\right)} \psi_{j, \ell}\left(\beta_{j}, \cdot\right)\right] \\ \quad+\frac{1}{2 \pi \mathrm{i}} \oint_{|z|=\exp (-\tau)} w(z, x) z^{n-1} \mathrm{~d} z, & n \geq 0 \\ \mathrm{i}\left[\sum_{j=1}^{Q} \sum_{\left(\mu_{j, \ell}\right)^{\prime}\left(\beta_{j}\right)<0} \frac{\exp \left(\mathrm{in} \beta_{j}\right)\left\langle f, \psi_{j, \ell}\left(\beta_{j}, \cdot\right)\right\rangle}{\left(\mu_{j, \ell}\right)^{\prime}\left(\beta_{j}\right)} \psi_{j, \ell}\left(\beta_{j}, \cdot\right)\right] & n<0 .\end{cases}
$$

\section{Numerical scheme}

In this section, we consider two numerical methods to approximate LAP solutions of (1)-(2). The first method (CCI-method) is based on the complex curve integral (25) and the second method (PM-method) is based on the propagating modes (28). Both cases involve numerical integral of solutions $\left.w(z, x)\right|_{\mathcal{C}_{0}}$ of the quasi-periodic problem (19)-(21) (or equivalently, the periodic problem (14)). As the quasi-periodic problems indexed by $z \mathrm{~s}$ are well-posed, they can be solved by classic numerical methods.

We apply the finite element method to solve the periodic problem (14). Suppose that $\Omega_{0}$ is covered by a family of regular and quasi-uniform meshes (see $\left.[32,33]\right) \mathcal{M}_{h}$ with the largest mesh size $h_{0}>0$. To construct periodic nodal functions, we suppose that the nodal points on the left and right boundaries have the same height. Omitting the nodal points on the right boundary, let $\left\{\phi_{M}^{(\ell)}: 1 \leq \ell \leq M\right\}$ be the piecewise linear nodal functions that equals to one at the $\ell$-th nodal and zero at other nodal points, then it is easily extended into a globally continuous and periodic function in $H_{l o c}^{1}(\Omega)$. $M$ is the number of nodal points which do not lie on the right boundary. Define the 
discretization subspace by:

$$
V_{h}:=\operatorname{span}\left\{\phi_{M}^{(1)}, \phi_{M}^{(2)}, \ldots, \phi_{M}^{(M)}\right\} \subset H_{p e r}^{1}\left(\Omega_{0}\right) .
$$

Thus we are looking for the finite element solution to (14) with the expansion

$$
v_{z}^{h}(x)=\sum_{\ell=1}^{M} c_{z}^{\ell} \phi_{m}^{(\ell)}(x)
$$

satisfies

$$
a_{z}\left(v_{z}^{h}, \phi_{h}\right)=-\int_{\Omega_{0}} z^{-x_{1}} f \overline{\phi_{h}} \mathrm{~d} x, \quad \forall \phi_{h} \in V_{h} .
$$

By Theorem 14 in [12] the finite element approximation is estimated as follows.

Theorem 3 Suppose $f \in L^{2}\left(\Omega_{0}\right)$ and $q \in W^{1, \infty}\left(\Omega_{0}\right)$, the solution $v_{z} \in H^{2}\left(\Omega_{0}\right)$ and the error between $v_{z}^{h}$ and $v_{z}$ is bounded by

$$
\left\|v_{z}^{h}-v_{z}\right\|_{L^{2}\left(\Omega_{0}\right)} \leq C h^{2}\|f\|_{L^{2}\left(\Omega_{0}\right)}, \quad\left\|v_{z}^{h}-v_{z}\right\|_{H_{p e r}^{1}\left(\Omega_{0}\right)} \leq C h\|f\|_{L^{2}\left(\Omega_{0}\right)}
$$

where $C>0$ is a constant independent of $z \in \mathcal{C}_{0}$. The estimations are also true for the function $w(z, x)=(\mathcal{F} u)(z, x)$ defined in Sect. 4.2 when $z \in \mathcal{C}_{0}$, i.e.,

$$
\begin{aligned}
& \left\|w_{h}(z, \cdot)-w(z, \cdot)\right\|_{L^{2}\left(\Omega_{0}\right)} \leq C h^{2}\|f\|_{L^{2}\left(\Omega_{0}\right)} \\
& \left\|w_{h}(z, \cdot)-w(z, \cdot)\right\|_{H_{p e r}^{1}\left(\Omega_{0}\right)} \leq C h\|f\|_{L^{2}\left(\Omega_{0}\right)} .
\end{aligned}
$$

In both methods, $w_{h}(z, \cdot)$ is the numerical approximation of the quasi-periodic solution $w(z, \cdot)$.

\subsection{CCl-method}

In this subsection, we approximate the LAP solution numerically with the help of the curve integral (25). $w(z, \cdot)$ is supposed to be known as it is easily computed by standard numerical methods. We can always choose different $n$ 's such that computations are carried out in different $\Omega_{n}$ 's, but in this section $n$ is taken to be 0 as an example. As the curve integral on $\mathbb{S}^{1}$ is standard, we only consider the case $k^{2} \in \sigma(A)$, so the LAP solution $u$ is written in the form of (25).

Recall that the number of different elements in $S_{+}^{0}$ is $Q$, and so is it in $S_{-}^{0}$. Thus $\mathbb{U F}=S_{+}^{0} \bigcup S_{-}^{0}$ has $2 Q$ elements when Assumption 2 holds. Especially, from Remark 3 and Assumption 2, neither 1 nor -1 lies in $\mathbb{U} \mathbb{F}$. Then the curve $\mathcal{C}_{0}$ is parameterized as follows.

Suppose $\mathbb{U F}=\left\{\varepsilon_{j}: j=1,2, \ldots, 2 Q\right\}$ and

$$
-\pi<\varepsilon_{1}<\varepsilon_{2}<\cdots<\varepsilon_{2 Q-1}<\varepsilon_{2 Q}<\pi .
$$


By assumption, for any $j=1, \ldots, 2 Q$, there is a sufficiently small $\delta>0$ such that the ball $B\left(\exp \left(\mathrm{i} \varepsilon_{j}\right), \delta\right)$ does not contain any other poles except $\exp \left(\mathrm{i} \varepsilon_{j}\right)$ itself. Let $\varepsilon_{j}^{-}<\varepsilon_{j}^{+}$be two angles such that $\left\{\exp \left(\mathrm{i} \varepsilon_{j}^{+}\right), \exp \left(\mathrm{i} \varepsilon_{j}^{-}\right)\right\}=\partial B\left(\exp \left(\mathrm{i} \varepsilon_{j}\right), \delta\right) \cap \mathbb{S}^{1}$. For sufficiently small $\delta>0$,

$$
-\pi<\varepsilon_{1}^{-}<\varepsilon_{1}<\varepsilon_{1}^{+}<\varepsilon_{2}^{-}<\varepsilon_{2}<\varepsilon_{2}^{+}<\cdots<\varepsilon_{2 Q}^{-}<\varepsilon_{2 Q}<\varepsilon_{2 Q}^{+}<\pi \text {. }
$$

Thus the curve segment of $\mathbb{S}^{1} \cap \mathcal{C}_{0}$ is parameterized as

$$
\mathcal{C}_{j}:=\left\{\exp (\mathrm{i} \varepsilon): \varepsilon \in\left[\varepsilon_{j}^{+}, \varepsilon_{j+1}^{-}\right]\right\}, \quad j=1,2, \ldots, 2 Q
$$

where $\varepsilon_{2 Q+1}^{-}:=\varepsilon_{1}^{-}+2 \pi$.

For any $j \in\{1,2, \ldots, 2 Q\}, \mathcal{C}_{0} \cap \partial B\left(\exp \left(\mathrm{i} \varepsilon_{j}\right), \delta\right)$ lies either in the exterior or in the interior of the unit disk, depending on $\epsilon_{j}$. As the curve segment is part of the circle with center $\exp \left(\mathrm{i} \epsilon_{j}\right)$, by choosing proper angles $\theta_{j}^{-}<\theta_{j}^{+}<\theta_{j}^{-}+2 \pi$. Thus the curve segment $\mathcal{C}_{0} \cap \partial B\left(\exp \left(i \varepsilon_{j}\right), \delta\right)$ is parameterized as

$$
\mathcal{D}_{j}=\left\{\exp \left(\mathrm{i} \varepsilon_{j}\right)+\delta \exp (\mathrm{i} \varepsilon), \quad \theta \in\left(\theta_{j}^{-}, \theta_{j}^{+}\right)\right\}, \quad j=1,2, \ldots, 2 Q .
$$

Now the whole curve $\mathcal{C}_{0}$ is parameterized piecewisely. Thus the representation of $u$ in (25) is written as

$$
\begin{aligned}
u(x)= & \frac{1}{2 \pi \mathrm{i}} \sum_{j=1}^{2 Q} \int_{\mathcal{C}_{j}} w(z, x) z^{-1} \mathrm{~d} z+\frac{1}{2 \pi \mathrm{i}} \sum_{j=1}^{2 Q} \int_{\mathcal{D}_{j}} w(z, x) z^{-1} \mathrm{~d} z \\
= & \frac{1}{2 \pi} \sum_{j=1}^{2 Q} \int_{\epsilon_{j}^{+}}^{\epsilon_{j+1}^{-}} w(\exp (\mathrm{i} t), x) \mathrm{d} t \\
& +\frac{1}{2 \pi} \sum_{j=1}^{2 Q} \int_{\theta_{j}^{-}}^{\theta_{j}^{+}} w\left(\exp \left(\mathrm{i} \alpha_{j}\right)+\delta \exp (\mathrm{i} t), x\right) \frac{\delta \exp (\mathrm{i} t)}{\exp \left(\mathrm{i} \alpha_{j}\right)+\delta \exp (\mathrm{i} t)} \mathrm{d} t
\end{aligned}
$$

Each integrand depends smoothly on $t$. Thus we only need to consider the numerical integration

$$
\mathcal{I}(g):=\int_{a}^{b} g(t, x) \mathrm{d} t,
$$

where $a<b$ and $g$ depends smoothly on $t \in[a, b]$ and $g(t, \cdot) \in H^{2}\left(\Omega_{0}\right)$ for any fixed $t$.

For an efficient numerical integral, we adopt the method introduced in [14], which comes originally from Sect. 9.6, [29]. Let $s(\tau):(a, b) \rightarrow[-\pi, \pi]$ be a function in $C^{\infty}(a, b)$ and strictly monotonically increasing function and satisfies

$$
s(-\pi)=a ; s(\pi)=b ; s^{(\ell)}(-\pi)=s^{(\ell)}(\pi)=0, \ell=1,2, \ldots, N_{0}
$$


for some positive integer $N_{0}$. Let $t=s(\tau)$, the integral $\mathcal{I}(g)$ becomes

$$
\mathcal{I}(g)=\int_{-\pi}^{\pi} g(s(\tau), x) s^{\prime}(\tau) \mathrm{d} \tau:=\int_{-\pi}^{\pi} h(\tau, x) \mathrm{d} \tau .
$$

Thus the new integrand $h$ depends smoothly on $\tau$ and is extended to a periodic function with respect to $\tau$.

We approximate the integral $\mathcal{I}(g)$ by trapezoidal rule. Let $[-\pi, \pi]$ be divided uniformly into $N$ subintervals, and the grid points be

$$
t_{j}=-\pi+\frac{2 \pi}{N} j, \quad j=1, \ldots, N
$$

The integral is approximated by

$$
\mathcal{I}_{N}(g)=\frac{2 \pi}{N} \sum_{\ell=1}^{N} h\left(t_{\ell}, x\right)=\frac{2 \pi}{N} \sum_{\ell=1}^{N} g\left(s\left(t_{\ell}\right), x\right) s^{\prime}\left(t_{\ell}\right)
$$

Then we recall the result of Theorem 9.33 in [29], and obtain the error estimation of the integral via (31):

$$
\left\|\mathcal{I}(g)-\mathcal{I}_{N}(g)\right\|_{H^{2}\left(\Omega_{0}\right)} \leq C N^{-N_{0}}\|s\|_{C^{N_{0}\left([-\pi, \pi] ; H^{2}\left(\Omega_{0}\right)\right)}} .
$$

We apply the quadrature rule (31) to approximate $u(x)$ :

$$
\begin{aligned}
u_{N}(x)= & \frac{2 \pi}{N} \sum_{j=1}^{2 Q}\left[\sum_{\ell=1}^{N} w\left(\exp \left(\mathrm{is}_{j}\left(t_{\ell}\right)\right), x\right) s_{j}^{\prime}\left(t_{\ell}\right)\right. \\
& \left.+\sum_{\ell=1}^{N} w\left(\exp \left(\mathrm{i} \alpha_{j}\right)+\delta \exp \left(\mathrm{is}_{j+2 Q}\left(t_{\ell}\right)\right), x\right) \frac{\delta s_{j+2 Q}^{\prime}\left(t_{\ell}\right) \exp \left(\mathrm{i} s_{j+2 Q}\left(t_{\ell}\right)\right)}{\exp \left(\mathrm{i} \alpha_{j}\right)+\delta \exp \left(\mathrm{i} s_{j+2 Q}\left(t_{\ell}\right)\right)}\right]
\end{aligned}
$$

where $s_{j}$ be the smooth function from $\left[\epsilon_{j}^{+}, \epsilon_{j+1}^{-}\right]$to $[-\pi, \pi]$ and $s_{j+2 Q}$ are the smooth function from $\left[\theta_{j}^{-}, \theta_{j}^{+}\right]$to $[-\pi, \pi]$. With (32), we conclude that

$$
\left\|u(x)-u_{N}(x)\right\|_{H^{2}\left(\Omega_{0}\right)} \leq C N^{-N_{0}+1 / 2}\|w\|_{C^{N_{0}\left([-\pi, \pi] ; H^{2}\left(\Omega_{0}\right)\right)}} .
$$

Finally, we replace $w(z, x)$ by the finite element solution $w_{h}(z, x)$. Then the LAP solution is approximated by 


$$
\begin{aligned}
u_{N, h}(x)= & \frac{1}{N} \sum_{j=1}^{2 Q}\left[\sum_{\ell=1}^{N} w_{h}\left(\exp \left(\mathrm{is}_{j}\left(t_{\ell}\right)\right), x\right) s_{j}^{\prime}\left(t_{\ell}\right)\right. \\
& \left.+\sum_{\ell=1}^{N} w_{h}\left(\exp \left(\mathrm{i} \epsilon_{j}\right)+\delta \exp \left(\mathrm{is}_{j}\left(t_{\ell}\right)\right), x\right) \frac{\delta s_{j+2 Q}^{\prime}\left(t_{\ell}\right) \exp \left(\mathrm{i} s_{j}\left(t_{\ell}\right)\right)}{\exp \left(\mathrm{i} \alpha_{j}\right)+\delta \exp \left(\mathrm{is}_{j}\left(t_{\ell}\right)\right)}\right] .
\end{aligned}
$$

Then we conclude the error between $u_{N, h}$ and $u$ in the domain $\Omega_{0}$.

Theorem 4 Let $u_{N, h}$ be the numerical LAP solution comes from the finite element method and the integral approximation (35). Then the error is bounded by

$$
\begin{aligned}
& \left\|u_{N, h}-u\right\|_{L^{2}\left(\Omega_{0}\right)} \leq C\left(N^{-N_{0}}+h^{2}\right)\|f\|_{L^{2}\left(\Omega_{0}\right)} ; \\
& \left\|u_{N, h}-u\right\|_{H^{1}\left(\Omega_{0}\right)} \leq C\left(N^{-N_{0}}+h\right)\|f\|_{L^{2}\left(\Omega_{0}\right)},
\end{aligned}
$$

where $C$ is a constant that depends on $Q$ and $N_{0}$, but does not depend on $N$ and $h$.

Proof From the representations of $u_{N, h}$ and $u$, and also the results from (34), we have the following error estimation:

$$
\begin{aligned}
& \left\|u_{N, h}-u\right\|_{L^{2}\left(\Omega_{0}\right)} \leq\left\|u_{N, h}-u_{N}\right\|_{L^{2}\left(\Omega_{0}\right)}+\left\|u_{N}-u\right\|_{L^{2}\left(\Omega_{0}\right)} \\
& \leq \| \frac{1}{N} \sum_{j=1}^{2 Q}\left[\sum_{\ell=1}^{N}\left(w_{h}-w\right)\left(\exp \left(\mathrm{i} s_{j}\left(t_{\ell}\right)\right), x\right) s_{j}^{\prime}\left(t_{\ell}\right)\right. \\
& \left.\quad+\sum_{\ell=1}^{N}\left(w-w_{h}\right)\left(\exp \left(\mathrm{i} \epsilon_{j}\right)+\delta \exp \left(\mathrm{i} s_{j}\left(t_{\ell}\right)\right), x\right) \frac{\delta s_{j+2 Q}^{\prime}\left(t_{\ell}\right) \exp \left(\mathrm{i} s_{j}\left(t_{\ell}\right)\right)}{\exp \left(\mathrm{i} \alpha_{j}\right)+\delta \exp \left(\mathrm{i} s_{j}\left(t_{\ell}\right)\right)}\right] \|_{L^{2}\left(\Omega_{0}\right)} \\
& \quad+C N^{-N_{0}}\|f\|_{H^{1}\left(\Omega_{0}\right)} \\
& \leq \frac{C}{N} \sum_{j=1}^{2 Q} \sum_{\ell=1}^{N}\left[\left\|\left(w-w_{h}\right)\left(\exp \left(\mathrm{i} \epsilon_{j}\right)+\delta \exp \left(\mathrm{i} s_{j}\left(t_{\ell}\right)\right), \cdot\right)\right\|_{L^{2}\left(\Omega_{0}\right)}\right. \\
& \left.\quad+\left\|\left(w-w_{h}\right)\left(\exp \left(\mathrm{i} \epsilon_{j}\right)+\delta \exp \left(\mathrm{i} s_{j}\left(t_{\ell}\right)\right), \cdot\right)\right\|_{L^{2}\left(\Omega_{0}\right)}\right]+C N^{-N_{0}}\|f\|_{H^{1}\left(\Omega_{0}\right)} \\
& \leq C
\end{aligned}
$$

The estimation of the $H^{1}$-norm is also obtained in the same way:

$$
\left\|u_{N, h}-u\right\|_{H^{1}\left(\Omega_{0}\right)} \leq C\left(h+N^{-N_{0}}\right)\|f\|_{H^{1}\left(\Omega_{0}\right)} .
$$

The proof is finished. 


\subsection{PM-method}

In this subsection, we introduce another numerical method based on (28). The integration part in (28) can be approximated in the same way as the CCI method, thus we only need to deal with the term expanded by eigenfunctions.

The first step is to find out explicit values of $\beta_{j}$ and corresponding eigenfunctions $\psi_{j, \ell}\left(\beta_{j}, \cdot\right)$. From the variational formulation (14) and by replacing $\log (z)$ by i $\alpha$ in (14), the problem is to find $\alpha \in(-\pi, \pi]$ such that there is a non-trival $v \in H_{p e r}^{1}\left(\Omega_{0}\right)$ solving

$$
\int_{\Omega_{0}}\left[\nabla v \cdot \nabla \bar{\phi}+\mathrm{i} \alpha\left(v \frac{\partial \bar{\phi}}{\partial x_{1}}-\frac{\partial v}{\partial x_{1}} \bar{\phi}\right)-\left(k^{2} q-\alpha^{2}\right) v \bar{\phi}\right] \mathrm{d} x=0
$$

for any $\phi \in H_{\text {per }}^{1}\left(\Omega_{0}\right)$. From Theorem 2.4, [3], it is written as a generalized eigenvalue problem:

$$
\left(\begin{array}{cc}
I-K_{0} & 0 \\
0 & I
\end{array}\right)\left(\begin{array}{l}
u_{1} \\
u_{2}
\end{array}\right)=-\alpha\left(\begin{array}{cc}
B & C^{1 / 2} \\
-C^{1 / 2} & 0
\end{array}\right)\left(\begin{array}{l}
u_{1} \\
u_{2}
\end{array}\right) .
$$

By solving this problem, we obtain the values of $\beta_{j}$ and the corresponding eigenfunctions $\psi_{j, \ell}\left(\beta_{j}, \cdot\right)$ at the same time. Then the values of $\left\langle f, \psi_{j, \ell}\left(\beta_{j}, \cdot\right)\right\rangle$ are easily calculated by numerical integral on triangular meshes.

The second step is to evaluate $\left(\mu_{j, \ell}\right)^{\prime}\left(\beta_{j}\right)$. A natural idea is to compute the derivative directly by a standard formula, such as the Newton's difference quotient or the symmetric difference quotient. However, since in these formulas, the denominators are always small and the numerators are not exact, the errors are expected to be larger. Due to this disadvantage, we use an alternative formula to compute $\left(\mu_{j, \ell}\right)^{\prime}\left(\beta_{j}\right)$.

First we need the definition of the energy flux:

$$
\mathcal{E}(\phi, \phi)=4 k \Im \int_{\Gamma_{j}} \frac{\partial \phi}{\partial x_{1}} \bar{\phi} \mathrm{d} s .
$$

The energy flux is closely related to the directions of propagating modes. When $\phi$ is propagating to the right, $\mathcal{E}(\phi ; \phi)>0$; while when it is propagating to the left, $\mathcal{E}(\phi ; \phi)<0$. Then we recall the following equation from the proof of Theorem 3 in [2]:

$$
\begin{aligned}
& \mu_{j, \ell}^{\prime}\left(\beta_{j}\right) \int_{\Omega_{0}} q(x) \psi_{j, \ell}\left(\beta_{j}, x\right) \overline{\psi_{j, \ell}\left(\beta_{j}, x\right)} \mathrm{d} x \\
& =\Im \int_{\Gamma_{j}} \frac{\partial \phi}{\partial x_{1}} \bar{\phi} \mathrm{d} s=\frac{E\left(\psi_{j, \ell}\left(\beta_{j}, \cdot\right), \psi_{j, \ell}\left(\beta_{j}, \cdot\right)\right)}{2 k} .
\end{aligned}
$$

Thus we have the following formula to compute the value of $\mu_{j, \ell}^{\prime}\left(\beta_{j}\right)$ :

$$
\mu_{j, \ell}^{\prime}\left(\beta_{j}\right)=\frac{E\left(\psi_{j, \ell}\left(\beta_{j}, \cdot\right), \psi_{j, \ell}\left(\beta_{j}, \cdot\right)\right)}{2 k \int_{\Omega_{0}} q(x) \psi_{j, \ell}\left(\beta_{j}, x\right) \overline{\psi_{j, \ell}\left(\beta_{j}, x\right)} \mathrm{d} x} .
$$


Fig. 5 Periodic waveguide

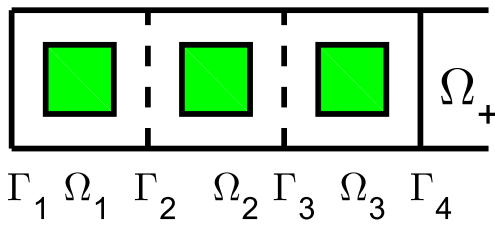

From the above formula the sign of $\mu_{j, \ell}^{\prime}\left(\beta_{j}\right)$ is exactly the same as that of the energy flux $E\left(\psi_{j, \ell}\left(\beta_{j}, \cdot\right), \psi_{j, \ell}\left(\beta_{j}, \cdot\right)\right)$.

Thus (28) is rewritten as:

$$
u\left(x_{1}+n, x_{2}\right)= \begin{cases}\mathrm{i}\left[\sum_{j=1}^{Q} \sum_{\ell=1}^{L_{j}} \nVdash_{j, \ell} \frac{\exp \left(\mathrm{i} n \beta_{j}\right)\left\langle f, \psi_{j, \ell}\left(\beta_{j}, \cdot\right)\right\rangle}{\left(\mu_{j, \ell}\right)^{\prime}\left(\beta_{j}\right)} \psi_{j, \ell}\left(\alpha_{j}, \cdot\right)\right] \\ +\frac{1}{2 \pi \mathrm{i}} \oint_{|z|=\exp (-\tau)} w(z, x) z^{n-1} \mathrm{~d} z, & n \geq 0 ; \\ \mathrm{i}\left[\sum_{j=1}^{Q} \sum_{\ell=1}^{L_{j}}\left(1-\nVdash_{j, \ell}\right) \frac{\exp \left(\mathrm{in} \alpha_{j}\right)\left\langle f, \psi_{j, \ell}\left(\beta_{j}, \cdot\right)\right\rangle}{\left(\mu_{j, \ell}\right)^{\prime}\left(\beta_{j}\right)} \psi_{j, \ell}\left(\beta_{j}, \cdot\right)\right. & n<0 ; \\ & +\frac{1}{2 \pi \mathrm{i}} \oint_{|z|=\exp (\tau)} w(z, x) z^{n-1} \mathrm{~d} z,\end{cases}
$$

where $\nVdash_{j, \ell}$ is an indicator function with the value 1 if $\mathcal{E}\left(\psi_{j, \ell}, \psi_{j, \ell}\right)>0$, and with the value 0 if $\mathcal{E}\left(\psi_{j, \ell}, \psi_{j, \ell}\right)<0$. We will show numerical results for the PM method also for wavenumbers that do not satisfy Assumption 2.

\section{Half-guide problems}

The numerical method introduced in this paper is also extended to half-guide problems. Let the half waveguide be defined by $\Omega_{+}:=\bigcup_{n=1}^{\infty} \Omega_{n}$ (see Fig. 5). Recall that

$$
\Omega_{0}=(-1 / 2,1 / 2] \times(0,1) ; \quad \Omega_{j}=\Omega_{0}+(j, 0)^{\top} .
$$

Then we are looking for the LAP solution $u_{+} \in H_{l o c}^{1}\left(\Omega_{+}\right)$such that it satisfies

$$
\Delta u_{+}+k^{2} q u_{+}=0 \quad \text { in } \Omega_{+} ; \quad u_{+}=\phi \quad \text { on } \Gamma_{1} .
$$

From [1], for any $\phi \in H^{1 / 2}\left(\Gamma_{1}\right)$ the LAP solution exists for all positive wavenumbers satisfying Assumption 1 except for a discrete set. In this section, we introduce numerical methods to approximate those LAP solutions with Assumptions 1 and 2. 


\subsection{Approximation of LAP solutions}

First, we define the following space:

$\mathcal{U}:=\left\{\left.u(f)\right|_{\Gamma_{1}}: u(f) \in H_{l o c}^{1}(\Omega)\right.$ is the LAP solution of $(1)-(2)$ with $\left.f \in L^{2}(\Omega)\right\}$.

From the analysis in [20], $\overline{\mathcal{U}}=H^{1 / 2}\left(\Gamma_{1}\right)$. For any fixed $\phi \in H^{1 / 2}\left(\Gamma_{1}\right)$, given any sufficiently small $\epsilon_{0}>0$, there is an $\Omega_{0}$-supported function $f$ such that $\left\|\left.u(f)\right|_{\Gamma_{1}}-\phi\right\|_{H^{1 / 2}\left(\Gamma_{1}\right)} \leq \epsilon_{0}$. Then $u_{+}$is approximated by:

$$
\tilde{u}_{+}\left(x_{1}+n, x_{2}\right)=\frac{1}{2 \pi \mathrm{i}} \oint_{\mathcal{C}_{0}} w(z, x) z^{n-1} \mathrm{~d} z, \quad x \in \Omega_{0}, n \in \mathbb{N}
$$

where $w(z, \cdot)$ solves (11)-(13) with the right hand side $f$, and $\mathcal{C}_{0}$ is defined by Definition 1 .

To solve this problem with the method based on the approximation (39), a two-step method is proposed. The first step is to determine a source term $f$ which approximates the boundary condition on $\Gamma_{1}$. The second step uses the source term to compute $u_{+}$, with the same method as the full-guide problem. As the second step is exactly the same as the full-guide problem, we only discuss the first step in this section.

Let $A$ be the operator from $L^{2}\left(\Omega_{0}\right)$ to $H^{1 / 2}\left(\Gamma_{1}\right)$ defined by

$$
\left.f \mapsto\left(\frac{1}{2 \pi \mathrm{i}} \oint_{\mathcal{C}_{0}} w(z, x) z^{-1} \mathrm{~d} z\right)\right|_{\Gamma_{1}}
$$

Since $u(f) \in H^{2}\left(\Omega_{0}\right), A f=u(f) \in H^{3 / 2}\left(\Gamma_{1}\right) \subset H^{1 / 2}\left(\Gamma_{1}\right)$. The compact embedding of $H^{3 / 2}\left(\Gamma_{1}\right)$ in $H^{1 / 2}\left(\Gamma_{1}\right)$ implies that $A$ is a compact operator with dense range. We are looking for an $f$ such that

$$
A f=\phi \quad \text { on } \Gamma_{1} \text {. }
$$

As the equation is severely ill-posed, we apply the well-known Tikhonov regularization method to find the "best solution" of this equation. For details we refer to Chapter 2.3 in [34].

\subsection{Numerical method}

Now we conclude the numerical scheme for half-guide scattering problems as follows.

1. Choose a family of basis functions for the space $L^{2}\left(\Omega_{0}\right)$ and denote it by $\left\{\phi_{\ell}\right\}_{\ell=1}^{\infty}$. Fix a sufficiently large integer $M_{0}>0$ and construct the matrix

$$
\Phi:=\left(A \phi_{1}, A \phi_{2}, \ldots, A \phi_{M_{0}}\right)
$$

We look for a best solution $c$ such that $\Phi c-\phi$ has the minimum value. 
2. Fix a small regularization parameter $\gamma>0$, compute the vector

$$
c(\gamma)=\left[\Phi^{*} \Phi+\gamma I\right]^{-1}\left(\Phi^{*} \phi\right)
$$

3. Approximate the function $f$ by $f_{\gamma}^{M_{0}}$ with coefficients $c(\gamma)$ :

$$
f_{\gamma}^{M_{0}}(x)=\sum_{\ell=1}^{M_{0}} c_{\ell}(\gamma) \phi_{\ell}(x)
$$

4. Solve the equation (19)-(21) with the source term $f_{\gamma}^{M_{0}}$. The LAP solution $u$ is then obtained by either the CCI method or the PM method.

From the convergence of the Tikhonov method and numerical solution of (39), this numerical scheme converges as $M_{0} \rightarrow \infty, \gamma \rightarrow 0, h \rightarrow 0$ and $N \rightarrow \infty$.

\section{Special wavenumbers}

\subsection{Formulation for special wavenumbers}

In previous sections, all discussions for the CCI method are based on Assumption 2, i.e., $P_{+} \cap P_{-}=\emptyset$. As it is not a necessary condition for the limiting absorption principle, we extend the CCI method to the case $P_{+} \cap P_{-} \neq \emptyset$ in this section. Assume that for the wavenumber $k_{0}>0$, Assumption 1 holds but Assumption 2 does not hold.

Following the notations in Sect. 5.3, $P=\left\{\beta_{1}, \ldots, \beta_{Q}\right\}$. Since $P_{+} \cap P_{-} \neq \varnothing$, assume that there is a positive integer $1 \leq Q^{\prime} \leq Q$ such that

$$
P_{+} \cap P_{-}=\left\{\beta_{1}, \ldots, \beta_{Q^{\prime}}\right\}
$$

For a visualization please refer to the right picture in Fig. 6. From (28), the solution can be written as finite number of propagation modes and a curve integral. If for some $j \in\{1,2, \ldots, Q\}, \mu_{j, \ell}^{\prime}\left(\beta_{j}\right)>0$ for all $\ell=1,2, \ldots, L_{j}$, then from [20],

$$
\mathrm{i} \sum_{\left(\mu_{j, \ell}\right)^{\prime}\left(\beta_{j}\right)>0} \frac{\exp \left(\mathrm{i} n \beta_{j}\right)\left\langle f, \psi_{j, \ell}\left(\beta_{j}, \cdot\right)\right\rangle}{\left(\mu_{j, \ell}\right)^{\prime}\left(\beta_{j}\right)} \psi_{j, \ell}\left(\beta_{j}, \cdot\right)=\frac{1}{2 \pi \mathrm{i}} \int_{\partial B\left(e^{\mathrm{i} \beta_{j}, \delta_{j}}\right)} w(z, x) z^{n-1} \mathrm{~d} z
$$

holds for all $n \in \mathbb{N}$ and sufficiently small $\delta_{j}>0$. In this case, we can merge this term with the curve integral in (28). Define the following domain:

$$
B_{\tau_{0}, \delta}=B\left(0, \exp \left(-\tau_{0}\right)\right) \bigcup\left[\bigcup_{\beta_{j} \in P^{+} \backslash P^{-}} B\left(e^{\mathrm{i} \beta_{j}}, \delta\right)\right] \backslash\left[\bigcup_{\beta_{j} \in P^{-} \backslash P^{+}} \overline{B\left(e^{\mathrm{i} \beta_{j}}, \delta\right)}\right]
$$



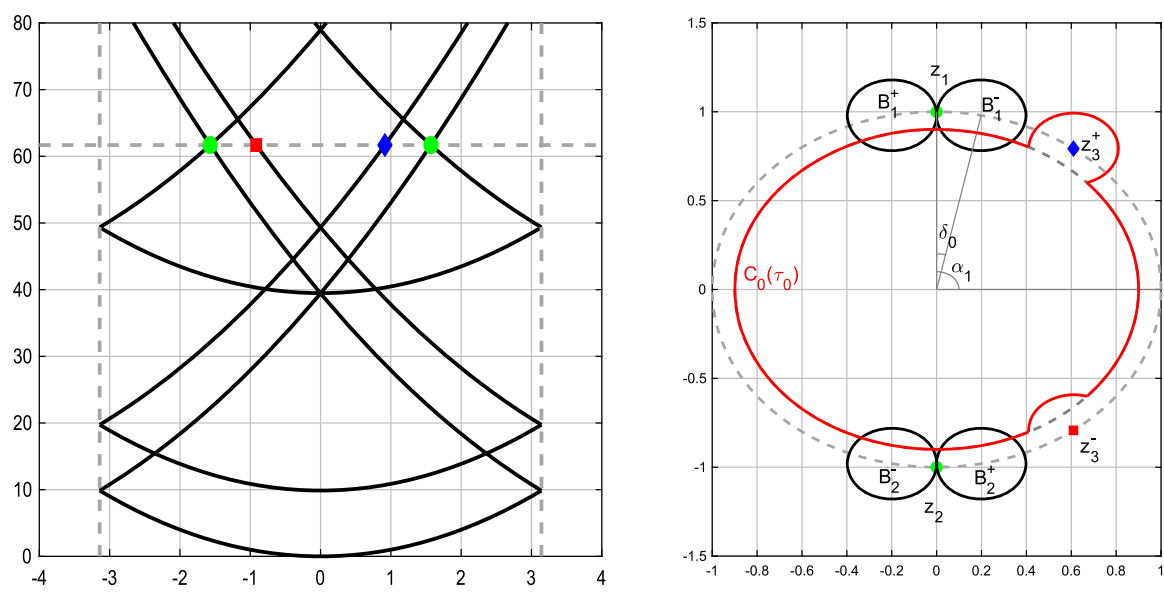

Fig. 6 Example for $n=1$ and $k_{0}^{2} \approx 61.685$. There are four unit Floquet multipliers, corresponding to $\alpha \approx \pm 1.5708, \pm 0.9151$. From the definition of $S_{0}^{ \pm}, P^{\prime}=2$ and $P=3$, the green circles are $z_{1} \approx$ $\exp (1.5708 \mathrm{i})$ and $z_{2} \approx \exp (-1.5708 \mathrm{i})$, the red square is $z_{3}^{-} \approx \exp (-0.9151 \mathrm{i})$ and the blue diamond is $z_{3}^{+} \approx \exp (0.9151 \mathrm{i})$. The red curve is $\mathcal{C}_{0}\left(\tau_{0}\right)$, the balls with black boundaries are $B_{j}^{ \pm}$where $j=1,2$

where $\delta>0$ satisfies all the conditions in Definition 1. For the visualization we refer to the red curve on the right picture in Fig. 6 . Then when $n \in \mathbb{N}$,

$$
\begin{aligned}
u\left(x_{1}+n, x_{2}\right)= & \mathrm{i}\left[\sum_{j=1}^{Q^{\prime}} \sum_{\left(\mu_{j, \ell}\right)^{\prime}\left(\beta_{j}\right)>0} \frac{\exp \left(\mathrm{in} \beta_{j}\right)\left\langle f, \psi_{j, \ell}\left(\beta_{j}, \cdot\right)\right\rangle}{\left(\mu_{j, \ell}\right)^{\prime}\left(\beta_{j}\right)} \psi_{j, \ell}\left(\beta_{j}, \cdot\right)\right] \\
& +\frac{1}{2 \pi \mathrm{i}} \oint_{\partial B_{\tau_{0}, \delta}} w(z, x) z^{n-1} \mathrm{~d} z,
\end{aligned}
$$

When $j=1,2, \ldots, Q^{\prime}$, since there are analytic functions $\mu_{j, \ell}$ passing through $\left(\beta_{j}, k_{0}^{2}\right)$ with both positive and negative values of $\mu_{j, \ell}^{\prime}\left(\beta_{j}\right)$, the integral on a small circle with center $e^{\mathrm{i} \beta_{j}}$ no longer works. Note that since $\mu_{j, \ell}$ depends analytically on $\alpha$ with non-vanishing derivatives at $\beta_{j}$, the inverse function, denoted by $\beta_{j, \ell}$, exists and depends analytically on $k^{2}$ (see Chapter 16, [27]). From inverse function theorem, when $\mu_{j, \ell}^{\prime}\left(\beta_{j}\right)>0, \beta_{j, \ell}\left(k^{2}\right)<\beta_{j}$ when $k^{2}<k_{0}^{2}$ and $\beta_{j, \ell}\left(k^{2}\right)>\beta_{j}$ when $k^{2}>k_{0}^{2}$. Similar for the case that $\mu_{j, \ell}^{\prime}\left(\beta_{j}\right)<0$. Moreover, when $k^{2} \neq k_{0}^{2}, \beta_{j, \ell}$ are separated for different $\ell$ thus the integral representation works in this case.

Here the balls $B_{j}^{ \pm}$are defined by:

$$
B_{j}^{ \pm}:=B\left(\exp \left[\mathrm{i}\left(\beta_{j} \pm \delta_{0}\right)\right], 2 \sin \left(\frac{\delta_{0}}{2}\right)\right)
$$

Furthermore, we suppose that $\delta_{0}$ is sufficiently small such that $\overline{B_{j}^{ \pm}} \cap S=\emptyset$. Please refer to the black circles in the right picture in Fig. 6. From above arguments, when 
$\mu_{j, \ell}^{\prime}\left(\beta_{j}\right)>0$

$$
e^{\mathrm{i} \beta_{j, \ell}\left(k^{2}\right)} \in B_{j}^{-} \text {when } k^{2}<k_{0}^{2} ; \quad e^{\mathrm{i} \beta_{j, \ell}\left(k^{2}\right)} \in B_{j}^{+} \text {when } k^{2}>k_{0}^{2}
$$

when $\mu_{j, \ell}^{\prime}\left(\beta_{j}\right)<0$,

$$
e^{\mathrm{i} \beta_{j, \ell}\left(k^{2}\right)} \in B_{j}^{+} \text {when } k^{2}<k_{0}^{2} ; \quad e^{\mathrm{i} \beta_{j, \ell}\left(k^{2}\right)} \in B_{j}^{-} \text {when } k^{2}>k_{0}^{2} ;
$$

For $j=1,2, \ldots, Q^{\prime}$, define $u_{j, k^{2}}^{+}$by:

$$
u_{j, k^{2}}^{+}(x):= \begin{cases}\frac{1}{2 \pi \mathrm{i}} \int_{\partial B_{j}^{-}} w_{k^{2}}(z, x) z^{-1} \mathrm{~d} z & \text { when } k^{2}<k_{0}^{2} \\ \frac{1}{2 \pi \mathrm{i}} \int_{\partial B_{j}^{+}} w_{k^{2}}(z, x) z^{-1} \mathrm{~d} z & \text { when } k^{2}>k_{0}^{2} .\end{cases}
$$

Then from a similar arguments in [20],

$$
u_{j, k^{2}}^{+}=\mathrm{i}\left[\sum_{\left(\mu_{j, \ell}\right)^{\prime}\left(\beta_{j}\right)>0} \frac{\exp \left(\mathrm{in} \beta_{j}\right)\left\langle f, \psi_{j, \ell}\left(\beta_{j}, \cdot\right)\right\rangle}{\left(\mu_{j, \ell}\right)^{\prime}\left(\beta_{j}\right)} \psi_{j, \ell}\left(\beta_{j}, \cdot\right)\right]
$$

holds uniformly when $k^{2}$ lies in a sufficiently small neighourhood of $k_{0}^{2}$. Thus

$$
u_{k^{2}}\left(x_{1}+n, x_{2}\right)=\frac{1}{2 \pi \mathrm{i}} \oint_{\partial B_{\tau_{0}, \delta}} w_{k^{2}}(z, x) z^{n-1} \mathrm{~d} z+\sum_{j=1}^{Q^{\prime}} u_{j, k^{2}}^{+}
$$

As this function depends real analytically on $k^{2}$, the LAP solution is the limit of (43) as $k^{2} \rightarrow k_{0}^{2}$, where the second term is defined in (42).

\subsection{Numerical method}

Now we introduce the numerical method based on (43). The first term with $k^{2}=k_{0}^{2}$ can be evaluated directly by the numerical method introduced in (33). For the second term, motivated by [5], an interpolation technique with respect to real valued $k^{2}$ is introduced to approximate the exact value. We conclude the algorithm as follows.

1. Compute the first term in (43) with $k^{2}=k_{0}^{2}$ from the method introduced in (35), denoted by $u_{0, N, h}$ with the same parameters $N$ and $h$.

2. Fix $M(M \geq 2)$ different wavenumbers $k_{m}^{2} \in K \backslash\left\{k_{0}^{2}\right\}, m=1,2, \ldots, M$, evaluate the values of $u_{k_{m}^{2}, j}^{+}$for $j=1,2, \ldots, Q^{\prime}$ in (43). The values are approximated by (42) with $N$ points, and denoted by $u_{j, k_{m}^{2}, N, h}^{+}$.

3. With these $M$ points, we approximate the value at $k_{0}^{2}$ by interpolation from $u_{j, k_{m}^{2}, N, h}^{+}$where $m=1,2, \ldots, M$. 
4. $u_{k_{0}^{2}, N, h}$ is then approximated by

$$
u_{k_{0}^{2}, N, M, h}\left(x_{1}+n, x_{2}\right)=u_{0, N, h}+\sum_{j=1}^{Q^{\prime}} u_{j, k_{0}^{2}, N, M, h}^{+}
$$

The following error estimations can be obtained in the same way as in Theorem 4:

$$
\begin{aligned}
\left\|u_{0, N, h}-u_{0}\right\|_{H^{\ell}\left(\Omega_{0}\right)}, & \left\|u_{j, k^{2}, N, h}^{+}-u_{j, k^{2}}^{+}\right\|_{H^{\ell}\left(\Omega_{0}\right)} \\
& \leq C\left(N^{-N_{0}}+h^{2-\ell}\right)\|f\|_{L^{2}\left(\Omega_{0}\right)}, \quad \ell=0,1 .
\end{aligned}
$$

Note that $C$ does not depend on $N$ and $h$, but it depends on $k^{2}$ and may blow up when $k^{2}$ is close to $k_{0}^{2}$. This is due to the asymptotic behavior of the poles when $k^{2} \rightarrow k_{0}^{2}$ : the poles approach $\exp \left(i \alpha_{j}\right)$ which lie on the integral contours $B_{j}^{ \pm}$. To obtain the error between $u_{k_{0}^{2}, N, h, M}$ and $u_{k_{0}^{2}}$, we only need to estimate the error from the interpolation. As $u_{j, k^{2}}^{+}$depends real analytically on $k^{2} \in K$, there is a point $K^{*} \in K$ such that

$$
u_{j, k^{2}}^{+}=\sum_{\ell=0}^{\infty} u_{\ell}^{j}\left(k^{2}-K^{*}\right)^{\ell}
$$

and there is a $C_{0}>0$ such that $\left\|u_{\ell}^{j}\right\|_{H^{1}\left(\Omega_{0}\right)} \leq C_{0}^{\ell}$ uniformly for $\ell=0,1,2, \ldots$ When we approximate $u_{j, k^{2}}^{+}$by interpolation, from standard error estimation of interpolation,

$$
\left\|u_{j, k_{0}^{2}, N, M, h}^{+}-u_{j, k_{0}^{2}}^{+}\right\|_{H^{1}\left(\Omega_{0}\right)} \leq C_{0}^{M}|K|^{M},
$$

where $|K|=\max _{x \neq y \in K}|x-y|$. Thus we can finally obtain the error estimation of the algorithm:

$$
\left\|u_{k_{0}^{2}, N, M, h}-u_{k_{0}^{2}}\right\|_{H^{\ell}\left(\Omega_{0}\right)} \leq C\left(N^{-N_{0}}+h^{2-\ell}+C_{0}^{M}|K|^{M}\right)\|f\|_{L^{2}\left(\Omega_{0}\right)},
$$

where $\ell=0,1$. However, in numerical results, the convergence rate of the third term is difficult to analyze. On one hand, to make sure that the Taylor expansion converges, we require that $|K|$ is sufficiently small; on the other hand, the error $\left\|u_{j, k^{2}, N, M, h}^{+}-u_{j, k^{2}}^{+}\right\|_{H^{\ell}\left(\Omega_{0}\right)}$ becomes larger when $k^{2} \rightarrow k_{0}^{2}$ due to the pole at $k_{0}^{2}$, as $C=C(|K|)$ may blow up. Moreover, $C_{0}$ can be a large number and it is impossible to be evaluated. 

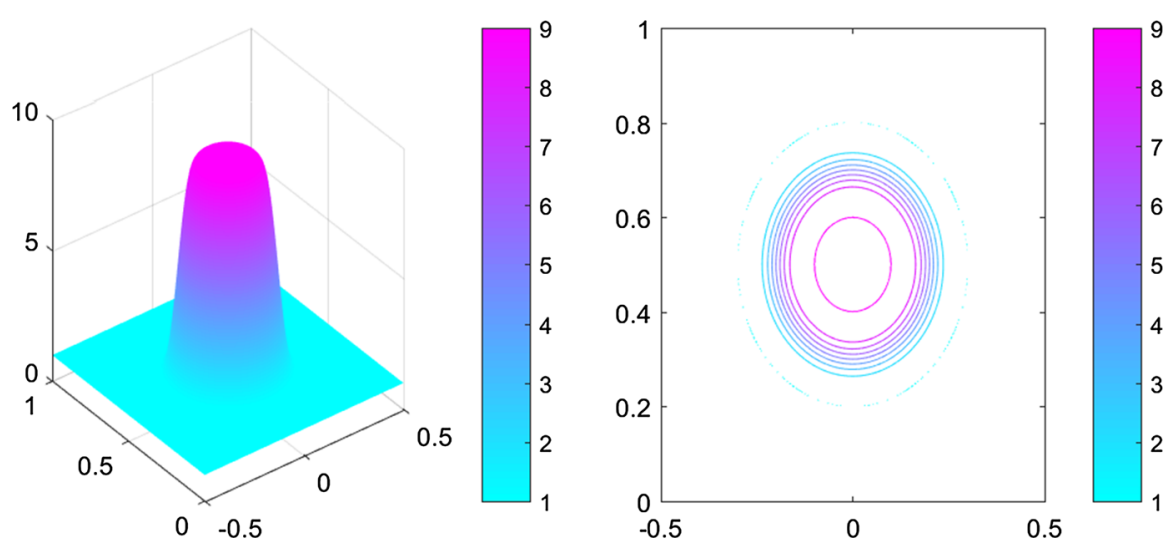

Fig. 7 Left: surface plot of $q$; right: contour plot of $q$

\section{Numerical results}

In this section, we present some numerical examples to show the efficiency of both the CCI-method and the PM-method. For all the examples, the function $q$ is chosen as follows (see Fig. 7):

$$
q(x)=\left\{\begin{array}{l}
1, \quad\left|x-a_{0}\right|>0.3 \\
9, \quad 0.1<\left|x-a_{0}\right|<0.3 \\
1+8 \zeta\left(\left|x-a_{0}\right|\right), \quad \text { otherwise }
\end{array}\right.
$$

The point $a_{0}=(0,0.5)$, and $\zeta(t)$ is a $C^{8}$-continuous function defined by

$\zeta(t)=\left\{\begin{array}{l}1, \quad t \leq a ; \\ 0, \quad t \geq b ; \\ 1-\left[\int_{\tau=a}^{b}(\tau-a)^{4}(\tau-b)^{4} \mathrm{~d} \tau\right]^{-1}\left[\int_{\tau=a}^{t}(\tau-a)^{4}(\tau-b)^{4} \mathrm{~d} \tau\right], a<t<b\end{array}\right.$

with $a=0.1, b=0.3$.

First, we draw the dispersion diagram and the corresponding $z$-space, see Fig. 8. From the dispersion diagram, we find out two stop bands, i.e., $(2.956 \pm 0.01,7.574 \pm$ $0.01)$ and $(13.41 \pm 0.01,15.49 \pm 0.01)$. When $k^{2}=5$, it lies in the first stop band, thus there is no eigenvalue on $\mathbb{S}^{1}$ in the $z$-space, i.e., $Q=0$. In this case, $u$ is represented by (18) with the integral on the unit circle $\mathbb{S}^{1}$. However, when $k^{2}=17$, there are two points lying on the dispersion curve with $\mu_{n}(\alpha)=17$. This implies that $Q=1$ and $\alpha_{1}^{+}=0.9576( \pm 0.01)$ (blue diamond) and $\alpha_{1}^{-}=-0.9576( \pm 0.01)$ (red square). Thus we design the integral curve in (25) as the red curve on the right. Moreover, we also check the condition number of the matrix obtained from the finite element discretization of (14), to find out a rough location of poles of the function $w(z, \cdot)$ (see Fig. 9). The parameter $N_{0}$ is fixed to be 6 for all the numerical examples in this section. 

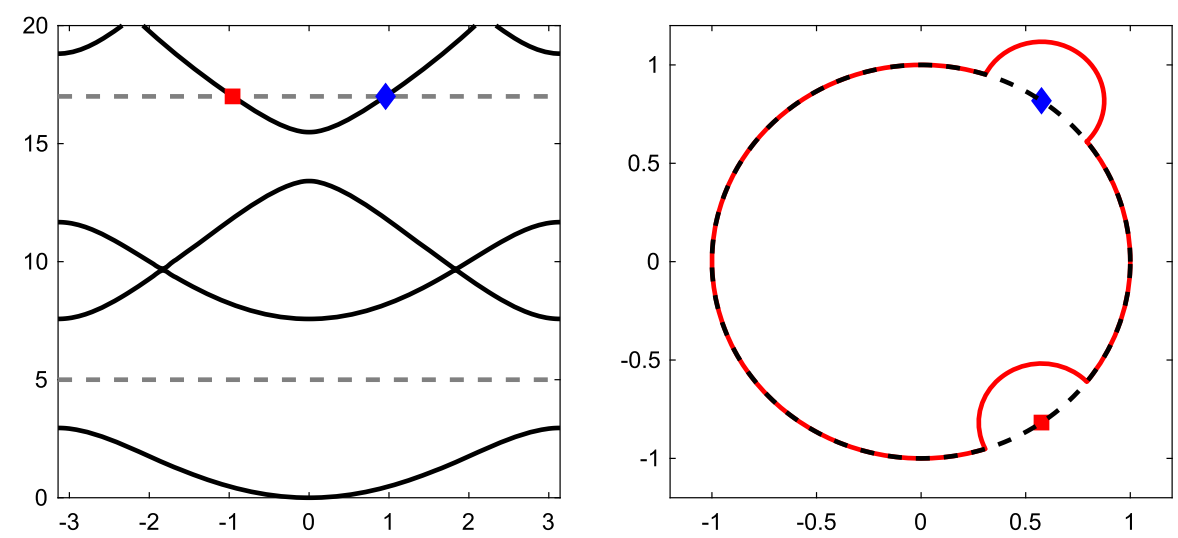

Fig. 8 Left: dispersion diagram; right: $z$-space

Fig. 9 Condition number on the complex plane

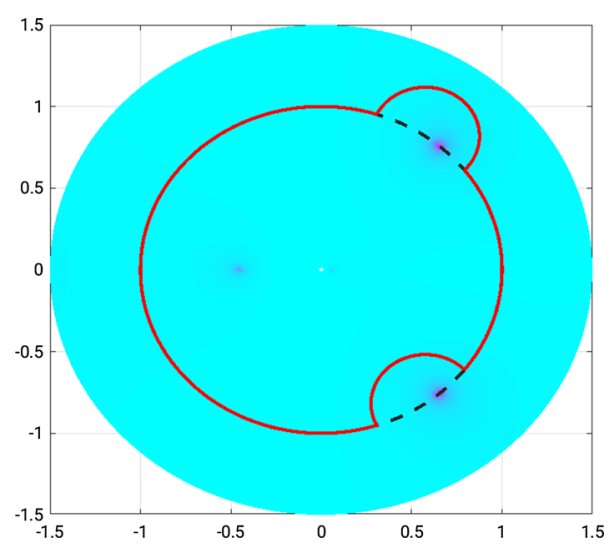

\subsection{Full-guide problems}

In the first part of this section, we show some numerical examples for scattering problems in full-waveguide, when Assumption 1 and 2 are both satisfied. The compactly supported source term $f$ is defined as follows:

$$
f(x)=\left\{\begin{array}{l}
0, \quad\left|x-a_{0}\right|>0.3 ; \\
3 \cos \left(2 \pi x_{1}\right) \sin \left(2 \pi x_{2}\right), \quad 0.1<\left|x-a_{0}\right|<0.3 \\
3 \zeta\left(\left|x-a_{0}\right|\right) \cos \left(2 \pi x_{1}\right) \sin \left(2 \pi x_{2}\right), \quad \text { otherwise }
\end{array}\right.
$$

where $a_{0}=(0.1,0.4)^{\top}$.

With these data, we calculate the value of $u$ for different parameters. For the finite element method, we choose $h=0.0025,0.005,0.01,0.02$ and $N=8,16,32,64$ for $k^{2}=5, N=4,8,16,32,64$ for $k^{2}=17$. We also compute "exact solutions" $u_{\text {exa }}$ from the finite element method introduced in $[5,6]$ with $h=0.005$ and the Lagrangian element. First we show the relative errors with different $h$ and $N$ for both cases, defined 
Table 1 Relative $L^{2}$-errors for $k^{2}=5$

\begin{tabular}{lllll}
\hline & $h=0.02$ & $h=0.01$ & $h=0.005$ & $h=0.0025$ \\
\hline$N=8$ & $2.46 \mathrm{E}-02$ & $2.37 \mathrm{E}-02$ & $2.34 \mathrm{E}-02$ & $2.33 \mathrm{E}-02$ \\
$N=16$ & $1.49 \mathrm{E}-03$ & $5.02 \mathrm{E}-04$ & $2.70 \mathrm{E}-04$ & $2.17 \mathrm{E}-04$ \\
$N=32$ & $1.34 \mathrm{E}-03$ & $3.40 \mathrm{E}-04$ & $9.86 \mathrm{E}-05$ & $5.64 \mathrm{E}-05$ \\
$N=64$ & $1.34 \mathrm{E}-03$ & $3.40 \mathrm{E}-04$ & $9.86 \mathrm{E}-05$ & $5.64 \mathrm{E}-05$ \\
\hline
\end{tabular}

Table 2 Relative $L^{2}$-errors for $k^{2}=17$-CCI-method

\begin{tabular}{lllll}
\hline & $h=0.02$ & $h=0.01$ & $h=0.005$ & $h=0.0025$ \\
\hline$N=4$ & $1.44 \mathrm{E}-01$ & $1.44 \mathrm{E}-01$ & $1.44 \mathrm{E}-01$ & $2.95 \mathrm{E}-02$ \\
$N=8$ & $3.05 \mathrm{E}-02$ & $2.97 \mathrm{E}-02$ & $2.95 \mathrm{E}-02$ & $2.95 \mathrm{E}-02$ \\
$N=16$ & $7.29 \mathrm{E}-03$ & $2.25 \mathrm{E}-03$ & $1.30 \mathrm{E}-03$ & $1.19 \mathrm{E}-03$ \\
$N=32$ & $7.22 \mathrm{E}-03$ & $1.98 \mathrm{E}-03$ & $8.06 \mathrm{E}-04$ & $6.37 \mathrm{E}-04$ \\
$N=64$ & $7.22 \mathrm{E}-03$ & $1.98 \mathrm{E}-03$ & $8.06 \mathrm{E}-04$ & $6.37 \mathrm{E}-04$ \\
\hline
\end{tabular}

Table 3 Relative $L^{2}$-errors for $k^{2}=17$-PM-method

\begin{tabular}{lllll}
\hline & $h=0.02$ & $h=0.01$ & $h=0.005$ & $h=0.0025$ \\
\hline$N=8$ & $2.14 \mathrm{E}-03$ & $2.17 \mathrm{E}-01$ & $2.17 \mathrm{E}-01$ & $2.17 \mathrm{E}-01$ \\
$N=16$ & $5.02 \mathrm{E}-02$ & $5.05 \mathrm{E}-02$ & $5.06 \mathrm{E}-02$ & $5.06 \mathrm{E}-02$ \\
$N=32$ & $7.78 \mathrm{E}-03$ & $4.94 \mathrm{E}-03$ & $4.72 \mathrm{E}-03$ & $4.71 \mathrm{E}-03$ \\
$N=64$ & $6.03 \mathrm{E}-03$ & $1.64 \mathrm{E}-03$ & $7.00 \mathrm{E}-04$ & $5.77 \mathrm{E}-04$ \\
\hline
\end{tabular}

by

$$
\operatorname{err}_{N, h}=\frac{\left\|u_{N, h}-u_{\text {exa }}\right\|_{L^{2}\left(\Omega_{0}\right)}}{\left\|u_{\text {exa }}\right\|_{L^{2}\left(\Omega_{0}\right)}}
$$

where $u_{N, h}$ is the numerical solution with parameter $N$ and $h$. Note that for $k^{2}=5$, the CCI-method and PM-method are the same, and the results are shown in Table 1. For $k^{2}=17$, the results from the CCI-method are shown in Table 2 and from the PM-method are in 3 . We can see that the relative error decays as $N$ gets larger and $h$ gets smaller. Note that when $N$ is sufficiently large (e.g., $N \geq 32$ ), the relative error does not decay when $N$ gets larger, this implies that the error brought by $N$ is relatively smaller, compared with the error from $h$. The decay rate of the CCI-method and the PM-method are relevant.

As the convergence rate with respect to $h$ is classical, we are especially interested in that of $N$. We fix $h=0.01$ for both cases, and compute the relative error between $u_{N, h}$ and $u_{256, h}$ for $k^{2}=5, u_{128, h}$ for $k^{2}=17$. From the result in (32), the error is expected to decay at the rate of $O\left(N^{-6}\right)$. From the two pictures in Fig. 10, the convergence is even faster than expected. 

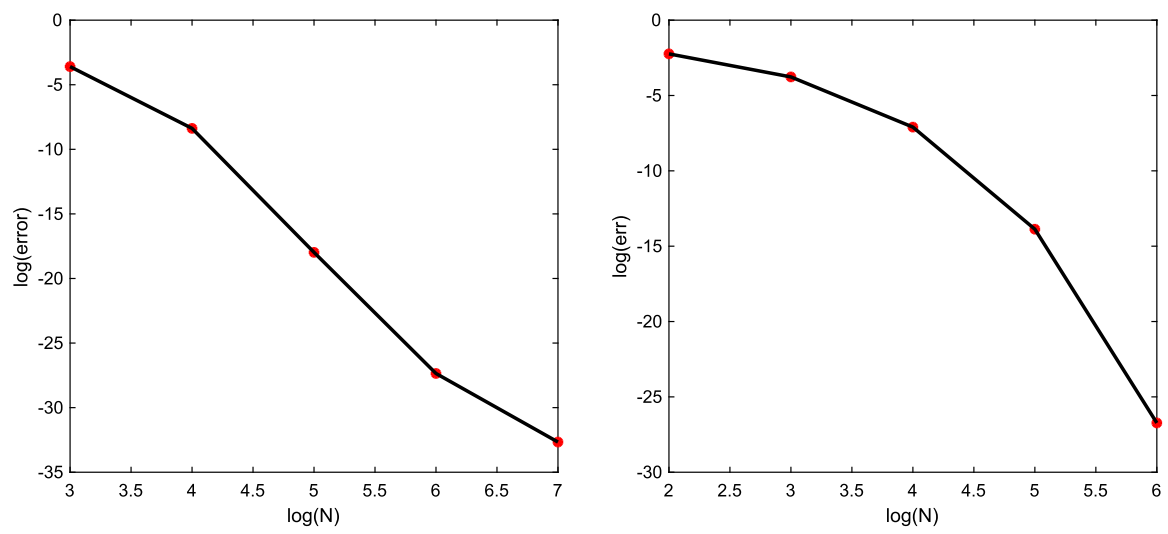

Fig. 10 Left: $k^{2}=5$; Right: $k^{2}=17$

We also compute the energy fluxes of propagating Bloch wave solutions for this example. We approximate the integrals

$$
u_{1}^{ \pm}=\frac{1}{2 \pi \mathrm{i}} \int_{\left|z-\exp \left(\mathrm{i} \alpha_{1}^{ \pm}\right)\right|=r} w(z, x) z^{-1} \mathrm{~d} z
$$

with the same method. Fix parameters $r=0.1$ and $N=16$, and the mesh size $h=0.005$. We obtain the values

$$
\mathcal{E}\left(u_{1}^{+}, u_{1}^{+}\right) \approx 0.00115, \quad \mathcal{E}\left(u_{1}^{-}, u_{1}^{-}\right) \approx-0.00115
$$

From above results, we conclude that $u_{1}^{+}$is propagating to the right, and $u_{1}^{-}$is propagating to the left according to the energy criteria, thus it coincides with the analysis in Sect. 6.3.

\subsection{Half-guide problems}

In the second part of this section, we show some numerical examples for half guide problems. The boundary data is given by

$$
\phi\left(x_{1}, x_{2}\right)=\sin x_{2}+0.5 x_{2}^{2}+\exp \left(\mathrm{i} x_{1}\right) \cos \left(2 x_{2}\right) \text {. }
$$

For all the examples, we approximate the source term by

$$
f_{\gamma}\left(x_{1}, x_{2}\right) \approx \sum_{j=-20}^{20} \sum_{\ell=0}^{10} \widehat{f}_{j, \ell}(\gamma) \exp \left(2 \mathrm{i} \pi j x_{1}\right) \cos \left(\pi \ell x_{2}\right)
$$

where $\gamma$ is the regularization parameter. As the numerical results also depend on $\gamma$, we show different results with respect to different regularization parameters, i.e., 

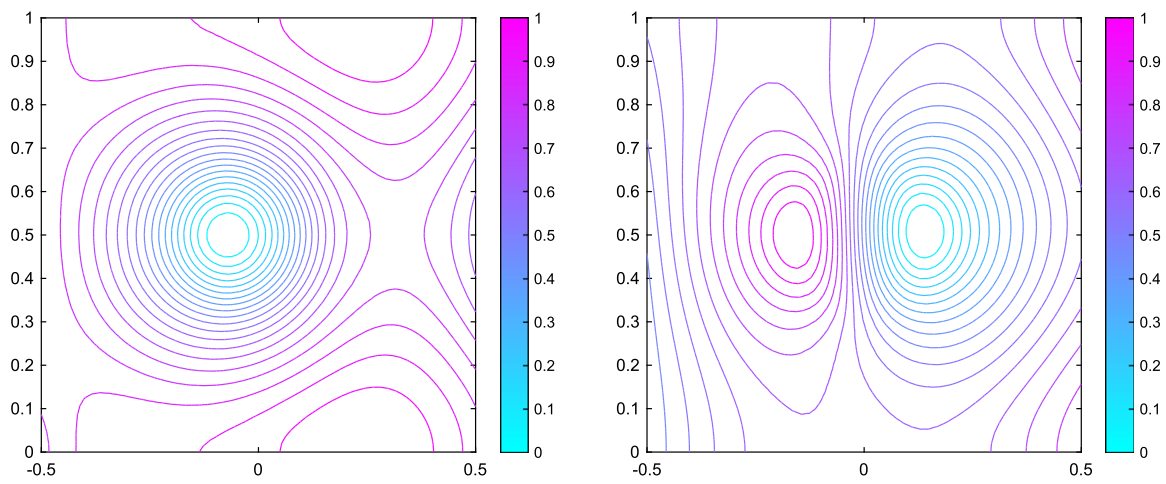

Fig. 11 Contour map of the solution with $k^{2}=17$. Left: real part of the solution; Right: imaginary part of the solution

Table 4 Relative $L^{2}$-errors for $k^{2}=5, \gamma=10^{-2}$

\begin{tabular}{llll}
\hline & $h=0.02$ & $h=0.01$ & $h=0.005$ \\
\hline$N=8$ & $4.24 \mathrm{E}-02$ & $3.96 \mathrm{E}-02$ & $3.86 \mathrm{E}-02$ \\
$N=16$ & $2.11 \mathrm{E}-02$ & $1.25 \mathrm{E}-02$ & $7.69 \mathrm{E}-03$ \\
$N=32$ & $2.10 \mathrm{E}-02$ & $1.25 \mathrm{E}-02$ & $7.66 \mathrm{E}-03$ \\
\hline
\end{tabular}

Table 5 Relative $L^{2}$-errors for $k^{2}=5, \gamma=10^{-5}$

\begin{tabular}{llll}
\hline & $h=0.02$ & $h=0.01$ & $h=0.005$ \\
\hline$N=8$ & $3.79 \mathrm{E}-02$ & $3.80 \mathrm{E}-02$ & $3.80 \mathrm{E}-02$ \\
$N=16$ & $3.38 \mathrm{E}-03$ & $9.53 \mathrm{E}-04$ & $4.49 \mathrm{E}-04$ \\
$N=32$ & $3.34 \mathrm{E}-03$ & $8.76 \mathrm{E}-04$ & $3.01 \mathrm{E}-04$ \\
\hline
\end{tabular}

$\gamma=10^{-2}$ and $10^{-5}$. The "exact solutions" also come from the method introduced in [5,6], and the relative error $\operatorname{err}_{N, h}$ is defined in the same way. For both cases, the numerical solutions are computed by the CCI-method. We show the results for $k^{2}=5$ with parameters $N=8,16,32$ and $h=0.02,0.01,0.005$ with $\gamma=10^{-2}$ in Table 4 and with $\gamma=10^{-5}$ in Table 5. For $k^{2}=17$, we show results for $N=4,8,16$ and $h=0.02,0.01,0.005$ with $\gamma=10^{-2}$ in Table 6 , and with $\gamma=10^{-5}$ in Table 7. We also give the contour map for the solution with $k^{2}=17, \gamma=10^{-5}, N=16$ and $h=0.05$ in Fig. 11. For all these cases, we can see that the error decays when $N$ gets larger (especially when $h$ is small) and $h$ gets smaller (especially when $N$ is large). However, the decaying rate slows down significantly when the parameter $N$ becomes sufficiently large (e.g., $N \geq 16$ ). This comes from the cut-off approximation of the series of $f$ and the regularization process. We also notice that the relative errors corresponding to $\gamma=10^{-2}$ is larger than that to $\gamma=10^{-5}$, which is also as expected. 
Table 6 Relative $L^{2}$-errors for $k^{2}=17, \gamma=10^{-2}$

\begin{tabular}{llll}
\hline & $h=0.02$ & $h=0.01$ & $h=0.005$ \\
\hline$N=4$ & $5.14 \mathrm{E}-02$ & $4.67 \mathrm{E}-02$ & $4.94 \mathrm{E}-02$ \\
$N=8$ & $4.11 \mathrm{E}-02$ & $2.09 \mathrm{E}-02$ & $1.17 \mathrm{E}-02$ \\
$N=16$ & $4.04 \mathrm{E}-02$ & $1.96 \mathrm{E}-02$ & $9.42 \mathrm{E}-03$ \\
\hline
\end{tabular}

Table 7 Relative $L^{2}$-errors for $k^{2}=17, \gamma=10^{-5}$

\begin{tabular}{llll}
\hline & $h=0.02$ & $h=0.01$ & $h=0.005$ \\
\hline$N=4$ & $6.18 \mathrm{E}-02$ & $5.68 \mathrm{E}-02$ & $5.58 \mathrm{E}-02$ \\
$N=8$ & $1.70 \mathrm{E}-02$ & $7.97 \mathrm{E}-03$ & $7.19 \mathrm{E}-03$ \\
$N=16$ & $1.55 \mathrm{E}-02$ & $3.81 \mathrm{E}-03$ & $1.93 \mathrm{E}-03$ \\
\hline
\end{tabular}
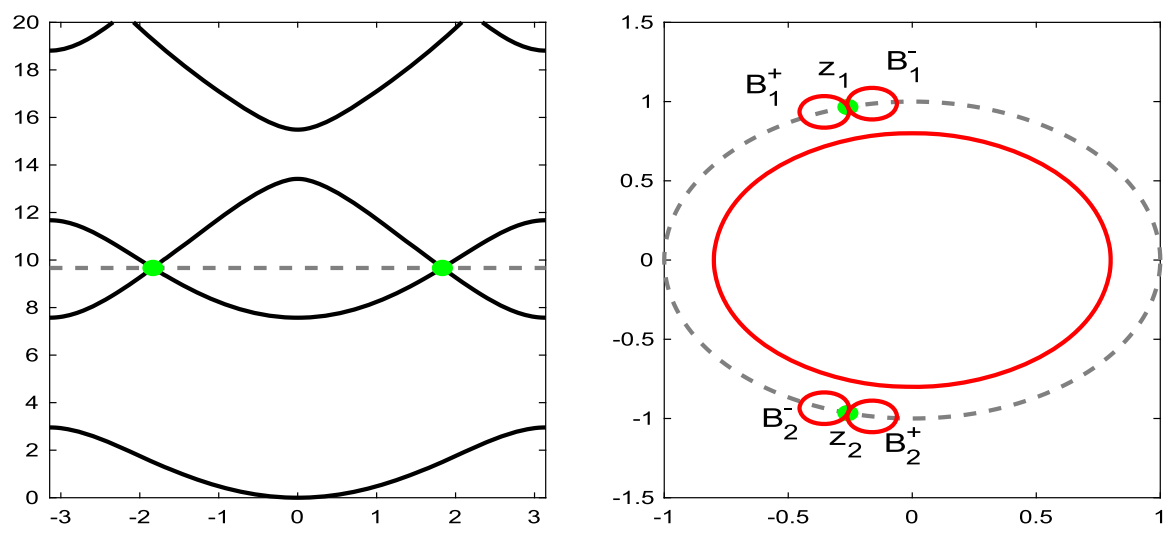

Fig. $12 k^{2}=9.6663( \pm 0.01)$. Left: dispersion diagram ; right: $z$-space

\subsection{Special wavenumbers}

In this section, we show some numerical results when Assumption 2 is not satisfied, i.e., $S_{+} \cap S_{-} \neq \emptyset$. From the dispersion diagram, i.e., Fig. 12, when $k^{2}=9.6663( \pm 0.01)$, Assumption 2 is not satisfied. However, we can not find the exact value of $k^{2}$ such that Assumption 2 is not satisfied, but a value very close to the exact one. Then the method introduced in Sect. 8 and the PM method is used for the numerical simulation. From the dispersion curve, $Q^{\prime}=Q=2, \alpha_{1}^{+}=\alpha_{2}^{-}=-1.8333( \pm 0.01)$ and $\alpha_{2}^{+}=$ $\alpha_{1}^{-}=1.8333( \pm 0.01)$. Let the curve $\mathcal{C}_{0}:=\left\{z \in \mathbb{C}^{2}:|z|=0.8\right\}$, and $B_{j}^{ \pm}(j=1,2)$ be defined by (41) with $\delta_{0}=0.1$. For the visualization of the points and curves we refer to Fig. 12.

For the CCI-method, we choose two different interpolation strategies to carry out the numerical approximation. For the first strategy, set $M=3$, and

$$
k_{1}^{2}=k^{2}-0.1, k_{2}^{2}=k^{2}+0.1, k_{3}^{2}=k^{2}+0.2 .
$$


Table 8 Relative $L^{2}$-errors for $k^{2}=9.6663( \pm 0.01)$ : CCI-method-strategy 1

\begin{tabular}{lllll}
\hline & $h=0.02$ & $h=0.01$ & $h=0.005$ & $h=0.0025$ \\
\hline$N=16$ & $2.98 \mathrm{E}-01$ & $3.00 \mathrm{E}-01$ & $3.01 \mathrm{E}-01$ & $3.01 \mathrm{E}-01$ \\
$N=32$ & $3.86 \mathrm{E}-02$ & $3.60 \mathrm{E}-02$ & $3.54 \mathrm{E}-02$ & $3.53 \mathrm{E}-02$ \\
$N=64$ & $3.60 \mathrm{E}-03$ & $2.40 \mathrm{E}-03$ & $2.27 \mathrm{E}-03$ & $2.25 \mathrm{E}-03$ \\
$N=128$ & $2.49 \mathrm{E}-03$ & $1.32 \mathrm{E}-03$ & $1.29 \mathrm{E}-03$ & $1.32 \mathrm{E}-03$ \\
\hline
\end{tabular}

Table 9 Relative $L^{2}$-errors for $k^{2}=9.6663( \pm 0.01)$ : CCI-method-strategy 2

\begin{tabular}{lllll}
\hline & $h=0.02$ & $h=0.01$ & $h=0.005$ & $h=0.0025$ \\
\hline$N=16$ & $2.85 \mathrm{E}-01$ & $2.87 \mathrm{E}-01$ & $2.87 \mathrm{E}-01$ & $2.87 \mathrm{E}-01$ \\
$N=32$ & $3.92 \mathrm{E}-02$ & $3.68 \mathrm{E}-02$ & $3.63 \mathrm{E}-02$ & $3.62 \mathrm{E}-02$ \\
$N=64$ & $3.59 \mathrm{E}-03$ & $2.39 \mathrm{E}-03$ & $2.25 \mathrm{E}-03$ & $2.24 \mathrm{E}-03$ \\
$N=128$ & $2.46 \mathrm{E}-03$ & $130 \mathrm{E}-03$ & $1.28 \mathrm{E}-03$ & $1.31 \mathrm{E}-03$ \\
\hline
\end{tabular}

Table 10 Relative $L^{2}$-errors for $k^{2}=9.6663( \pm 0.01)$ : PM-method

\begin{tabular}{lllll}
\hline & $h=0.006$ & $h=0.003$ & $h=0.0015$ & $h=0.00075$ \\
\hline$N=16$ & $1.42 \mathrm{E}-01$ & $1.42 \mathrm{E}-01$ & $1.42 \mathrm{E}-01$ & $1.42 \mathrm{E}-01$ \\
$N=32$ & $5.14 \mathrm{E}-03$ & $5.26 \mathrm{E}-03$ & $5.19 \mathrm{E}-03$ & $5.15 \mathrm{E}-03$ \\
$N=64$ & $1.47 \mathrm{E}-03$ & $1.72 \mathrm{E}-03$ & $1.49 \mathrm{E}-03$ & $1.35 \mathrm{E}-03$ \\
$N=128$ & $1.43 \mathrm{E}-03$ & $1.70 \mathrm{E}-03$ & $1.47 \mathrm{E}-03$ & $1.33 \mathrm{E}-03$ \\
\hline
\end{tabular}

For the second one, $M=5$ and

$$
k_{1}^{2}=k^{2}-0.2, k_{2}^{2}=k^{2}-0.1, k_{3}^{2}=k^{2}+0.1, k_{4}^{2}=k^{2}+0.2, k_{5}^{2}=k^{2}+0.3 .
$$

We still use the result obtained by the method introduced in [5,6] to produce the "exact solution", and compute the relative errors with the parameters $h=$ $0.02,0.01,0.005,0.0025$ and $N=16,32,64,128$. The results are shown in Table 8-9. In both tables, the relative errors with these two different strategies are similar, and the error decays when $N$ gets larger and $h$ gets smaller. These results show that the CCI-method for special numbers is convergent.

We also used the PM-method to approximate the LAP solution with this special wavenumber. We choose parameters $h=0.006,0.003,0.0015,0.00075$ and $N=$ $16,32,64,128$, and the results are shown in Table 10. From the results, the relative errors decay as $N$ gets larger and $h$ gets smaller, but the decay is not as stable as the CCI-method.

We also check the energy fluxes corresponding to the propagating modes. The approximation is carried out with the help of the second strategy, with parameters $h=0.005$ and $N=64$. The energy fluxes corresponding to $u_{1}^{ \pm}$and $u_{2}^{ \pm}$are evaluated 
as follows:

$$
\begin{aligned}
& \mathcal{E}\left(u_{1}^{+}, u_{1}^{+}\right) \approx 0.00235, \quad \mathcal{E}\left(u_{1}^{-}, u_{1}^{-}\right) \approx-0.00235, \\
& \mathcal{E}\left(u_{2}^{+}, u_{2}^{+}\right) \approx 0.0428, \quad \mathcal{E}\left(u_{2}^{-}, u_{2}^{-}\right) \approx-0.0428 .
\end{aligned}
$$

From the values, $u_{1}^{+}$and $u_{2}^{+}$are propagating to the right, $u_{1}^{-}$and $u_{2}^{-}$are propagating to the left. This coincides with the results shown in Sect. 6.2.

\subsection{Conclusion}

Now we compare the two different methods - the CCI-method and the PM-method. The CCI-method depends on a simplified integral representation (25) for the LAP solution with Assumption 1, where a suitable complex integral curve is to be designed specially depending on the behaviour of the Floquet multipliers with respect to the absorbing parameter $\epsilon$. Then the LAP solution is approximated by the sum of finite number of solutions of quasi-periodic problems, which are all well-posed. However, to know the behaviour of the poles, for example by producing a dispersion diagram, may take a relatively longer time. When Assumption 1 no longer holds, an interpolation technique is introduced to make the CCI-method suitable for this situation. This makes the CCI-method more complicated. But this method does not require very accurate evaluations of the exceptional values and Bloch wave solution. On the other hand, the PM-method is based on the curve integral with finite number of non-selfadjoint eigenvalue problem. Compared to the CCI-method, it does not depend on Assumption 1. We can decide if a Floquet mode is acceptable by the sign of the energy flux, so we do not need to know the dispersion curve in principle. However, as the non-selfadjoint eigenvalue problems have complex eigenvalues, sometimes it may not be easy to find out all real eigenvalues in $(-\pi, \pi]$. Moreover, the errors in computing eigenvalues, eigenfunctions and derivatives of the dispersion curves are relatively larger, thus we need a much finer mesh to have a solution with similar accuracy of solutions from the CCI-method. Note that for the full waveguide problem with $k=\sqrt{17}$, the results from the PM-method is relevant to the CCI-method. The reason may be the almost orthogonality between $f$ and the eigenfunctions.

We also compare the methods introduced in this paper with other methods. The computational complexity of both methods are equivalent to that of [14]. The problems are different, for the Floquet-Bloch transformed field has finite number of poles in this paper, but one or two branch cuts in [14]. The methods introduced in $[8,9,15]$ are based on the numerical evaluation of the DtN maps, which are described by the quadratic characteristic equation. The evaluations are carried out by the iteration method based on the cell problem, thus this may involve many times of the solutions of quasi-periodic problem. Another interesting method is introduced in [18], by approximating the LAP solution by finite number of propagating modes and a truncated problem. Suppose the truncated problem exists in the cells from $-N$ to $N$, and the degree of freedom is $M$ in one cell, then the degree of freedom for the whole problem is greater than $N M$. Thus they have to solve a system of at least $N M \times N M$. However, for our problem we only need to solve several times $M \times M$ linear system, which is much more efficient. Due 
to the super algebraic convergence, we do not need to solve the $M \times M$ linear system too many times. Thus our method is faster than the one introduced in that paper.

Acknowledgements This work is supported by the Deutsche Forschungsgemeinschaft (DFG, German Research Foundation) Project-ID 258734477 SFB 1173 and Project-ID 433126998. The author is grateful for Prof. Andreas Kirsch and two anonymous reviewers for the valuable suggestions to improve this paper.

Funding Open Access funding enabled and organized by Projekt DEAL.

Open Access This article is licensed under a Creative Commons Attribution 4.0 International License, which permits use, sharing, adaptation, distribution and reproduction in any medium or format, as long as you give appropriate credit to the original author(s) and the source, provide a link to the Creative Commons licence, and indicate if changes were made. The images or other third party material in this article are included in the article's Creative Commons licence, unless indicated otherwise in a credit line to the material. If material is not included in the article's Creative Commons licence and your intended use is not permitted by statutory regulation or exceeds the permitted use, you will need to obtain permission directly from the copyright holder. To view a copy of this licence, visit http://creativecommons.org/licenses/by/4.0/.

\section{Appendix}

Proof of Lemma 1 Assume that the set has a bounded accumulation point $k_{0} \in \mathbb{R}_{+}$, i.e., there is a sequence $k_{n}$ such that

$$
P_{+}\left(k_{n}\right) \cap P_{-}\left(k_{n}\right) \neq \emptyset, \quad \lim _{n \rightarrow \infty} k_{n}=k_{0} .
$$

Thus the sequence has a strictly monotones subsequence which also converges to $k_{0}$. Without loss of generality, we assume that the subsequence is monotonically decreasing and is still denoted by $k_{n}$, i.e.,

$$
k_{0}^{2}<\cdots<k_{n}^{2}<k_{n-1}^{2}<\cdots<k_{1}^{2} .
$$

This implies that for any $n \in \mathbb{N}$, there is a pair $\left(i_{n}, j_{n}\right) \in \mathbb{N} \times \mathbb{N}$ such that

$$
\exists \alpha_{n} \in(-\pi, \pi], \text { s.t., } \mu_{i_{n}}\left(\alpha_{n}\right)=\mu_{j_{n}}\left(\alpha_{n}\right)=k_{n}^{2}
$$

that satisfies

$$
\mu_{i_{n}}^{\prime}\left(\alpha_{n}\right)>0, \quad \mu_{j_{n}}^{\prime}\left(\alpha_{n}\right)<0 .
$$

As $\lim _{n \rightarrow \infty} \mu_{n}(\alpha)=\infty$ for any $\alpha \in(-\pi, \pi]$, there should be a subsequence of pairs $\left(i_{n}, j_{n}\right)$ such that $i_{n}=i_{0}$ and $j_{n}=j_{0}$ where $i_{0}$ and $j_{0}$ are two constant positive integers. Still denote the subsequence of $k_{n}^{2}$ by $k_{n}^{2}$, then for any $n \in \mathbb{N}$, there is an $\alpha_{n} \in(-\pi, \pi]$ such that

$$
\mu_{i_{0}}\left(\alpha_{n}\right)=\mu_{j_{0}}\left(\alpha_{n}\right)=k_{n}^{2}, \quad \mu_{i_{0}}^{\prime}\left(\alpha_{n}\right)>0, \mu_{j_{0}}^{\prime}\left(\alpha_{n}\right)<0 .
$$

Define the function

$$
\mu(\alpha):=\mu_{i_{0}}(\alpha)-\mu_{j_{0}}(\alpha)
$$


then there is a sequence $\alpha_{n} \in(-\pi, \pi]$ such that

$$
\mu\left(\alpha_{n}\right)=0, \quad \forall n \in \mathbb{N}
$$

As $\mu_{i_{0}}$ and $\mu_{j_{0}}$ are both analytic functions, $\mu$ is analytic as well. Thus either $\mu$ is a constant function equals to 0 , or $\alpha_{n}=\alpha_{0}$ except for a finite number of $n$ 's.

For the first case, $\mu^{\prime}(\alpha)=0$ for any $\alpha_{n}$, which contradicts with

$$
\mu^{\prime}\left(\alpha_{n}\right)=\mu_{i_{0}}^{\prime}\left(\alpha_{n}\right)-\mu_{j_{0}}^{\prime}\left(\alpha_{n}\right)>0 .
$$

For the second case, suppose there is an $N>>1$ such that $\alpha_{n}=\alpha_{0}$ for any $n \geq N$, then $\mu_{i_{0}}\left(\alpha_{n}\right)=\mu_{j_{0}}\left(\alpha_{n}\right)=k_{n}^{2}$ implies that $k_{n}^{2}=k_{0}^{2}$ for any $n \geq N$. This contradicts with the monotone decreasing property. Thus $k_{0}^{2}$ can not be an accumulation point, the proof is finished.

\section{References}

1. Hoang, V.: The limiting absorption principle for a periodic semin-infinite waveguide. SIAM J. Appl. Math. 71(3), 791-810 (2011)

2. Fliss, S., Joly, P.: Solutions of the time-harmonic wave equation in periodic waveguides: asymptotic behaviour and radiation condition. Arch. Rational Mech. Anal. 219(1), 349-386 (2016)

3. Kirsch, A., Lechleiter, A.: A radiation condition arising from the limiting absorption principle for a closed full- or half-waveguide problem. Math. Meth. Appl. Sci. 41(10), 3955-3975 (2018)

4. Kirsch, A., Lechleiter, A.: The limiting absorption principle and a radiation condition for the scattering by a periodic layer. SIAM J. Math. Anal. 50(3), 2536-2565 (2018)

5. Ehrhardt, M., Sun, J., Zheng, C.: Evaluation of scattering operators for semi-infinite periodic arrays. Commun. Math. Sci. 7(2), 347-364 (2009)

6. Ehrhardt, M., Han, H., Zheng, C.: Numerical simulation of waves in periodic structures. Commun. Comput. Phys. 5(5), 849-870 (2009)

7. Sun, J., Zheng, C.: Numerical scattering analysis of TE plane waves by a metallic diffraction grating with local defects. J. Opt. Soc. Am. A 26(1), 156-162 (2009)

8. Joly, P., Li, J.R., Fliss, S.: Exact boundary conditions for periodic waveguides containing a local perturbation. Commun. Comput. Phys. 1(6), 945-973 (2006)

9. Coatléven, J.: Helmholtz equation in periodic media with a line defect. J. Comput. Phys. 231, 16751704 (2012)

10. Haddar, H., Nguyen, T.P.: A volume integral method for solving scattering problems from locally perturbed infinite periodic layers. Appl. Anal. 96(1), 130-158 (2016)

11. Lechleiter, A.: The Floquet-Bloch Transform and Scattering from Locally Perturbed Periodic Surfaces. J. Math. Anal. Appl. 446(1), 605-627 (2017)

12. Lechleiter, A., Zhang, R.: A convergent numerical scheme for scattering of aperiodic waves from periodic surfaces based on the Floquet-Bloch transform. SIAM J. Numer. Anal. 55(2), 713-736 (2017)

13. Lechleiter, A., Zhang, R.: A Floquet-Bloch transform based numerical method for scattering from locally perturbed periodic surfaces. SIAM J. Sci. Comput. 39(5), B819-B839 (2017)

14. Zhang, R.: A High Order Numerical Method for Scattering from Locally Perturbed Periodic Surfaces. SIAM J. Sci. Comput. 40(4), A2286-A2314 (2018)

15. Fliss, S., Joly, P.: Exact boundary conditions for time-harmonic wave propagation in locally perturbed periodic media. Appl. Numer. Math. 59(9), 2155-2178 (2009)

16. Lecamp, G., Hugonin, J.P., Lalanne, P.: Theoretical and computational concepts for periodic optical waveguides. Optics Expr. 15(18), 11042-11060 (2007)

17. Alcázar-López, A., Méndez-Bernúdez, J.A., Luna-Acosta, G.A.: An efficient method to compute the scattering properties of long periodic waveguides, Journal of Physics: Conference Series, 4th National Meeting in Chaos. Complex Syst. Time Series 475, 012001 (2013) 
18. Dohnal, T., Schweizer, B.: A Bloch wave numerical scheme for scattering problems in periodic waveguides. SIAM J. Numer. Anal. 56(3), 1848-1870 (2018)

19. Fliss, S., Joly, P.: Wave propagation in locally perturbed periodic media(case with absorption): Numerical aspects. J. Comput. Phys. 231, 1244-1271 (2012)

20. Zhang, R.: Spectrum decomposition of translation operators in periodic waveguide. Preprint. https:// arxiv.org/pdf/1905.11091.pdf

21. Kuchment, P.: An overview of periodic elliptic operators. B. Am. Math. Soc. 53(3), 343-414 (2016)

22. Steinberg, S.: Meromorphic families of compact operators. Arch. Ration. Mech. Anal. 31(5), 372-379 (1968)

23. Yuan, L., Lu, Y.Y.: An efficient bidirectional propagation method based on Dirichlet-to-Neumann maps. IEEE Photon. Technol. Lett. 18(18), 1967-1969 (2006)

24. Ehrhardt, M., Zheng, C.: Fast numerical methods for waves in periodic media, Progress in Computational Physics (PiCP), 135-166, (2010)

25. Kuchment, P.: Floquet theory for partial differential equations, operator theory. Advances and Applications (60). Birkhäuser, Basel (1993)

26. Reed, M., Simon, B.: Methods of Modern Mathematical Physics. I. Functional Analysis. Academic Press, New York (1980)

27. Reed, M., Simon, B.: Methods of Modern Mathematical Physics. IV. Analysis of Operators. Academic Press, New York (1978)

28. Kato, T.: Perturbation Theory for Linear Operators. Springer, Berlin, Heidelberg (1995)

29. Kress, R.: Numerical Analysis. Springer, Berlin Heidelberg (1998)

30. Colton, D.L., Kress, R.: Inverse Acoustic and Electromagnetic Scattering Theory. Springer, Berlin Heidelberg (1998)

31. Atkinson, K.E.: An Introduction to Numerical Analysis. John Wiley \& Sons, Hoboken (1989)

32. Brenner, S.C., Scott, L.R.: The Mathematical Theory of Finite Element Methods. Springer, New York (1994)

33. Sauter, S., Schwab, C.: Boundary Element Methods. Springer, Berlin-New York (2007)

34. Cakoni, F., Colton, D.: Qualitative Methods in Inverse Scattering Theory. An Introduction. Springer, Berlin (2006)

Publisher's Note Springer Nature remains neutral with regard to jurisdictional claims in published maps and institutional affiliations. 